\title{
Vortex-induced Vibration of a 5:1 Rectangular Cylinder A comparison of wind tunnel sectional model tests and computational simulations
}

\author{
Dinh Tung Nguyen ${ }^{\mathrm{a}}$, David M. Hargreaves ${ }^{\mathrm{a}}$, John S. Owen ${ }^{\mathrm{a}}$ \\ ${ }^{a}$ Faculty of Engineering, University of Nottingham, UK
}

\begin{abstract}
Considered to be representative of a generic bridge deck geometry and characterised by a highly unsteady flow field, the 5:1 rectangular cylinder has been the main case study in a number of studies including the "Benchmark on the Aerodynamics of a Rectangular 5:1 Cylinder" (BARC). There are still a number of limitations in the knowledge of (i) the mechanism of the vortex-induced vibration (VIV) and (ii) of the turbulence-induced effect for this particular geometry. Extended computational and wind tunnel studies were therefore conducted by the authors to address these issues. This paper primarily describes wind tunnel and computational studies using a sectional model in an attempt to bring more insight into Point (i). By analysing the distribution and correlation of the surface pressure around an elastically mounted 5:1 rectangular cylinders in smooth and turbulent flow, it revealed that the VIV was triggered by the motion-induced leading-edge vortex; a strongly correlated flow feature close to the trailing edge was then responsible for an increase in the structural response.
\end{abstract}

Keywords: 5:1 rectangular cylinder, BARC, vortex-induced vibration, turbulent flow, wind tunnel, LES simulation

\section{1. Introduction}

2 The rectangular cylinder has been considered as representative of many structures in 3 the built environment including the bridge deck. In contrast to the circular cylinder, the 4 rectangular cylinder is characterised by permanent separation points at the leading edge 5 causing two unstable shear layers which can interact with the after-body length or with each 6 other in the wake, significantly affecting its response (Nakamura et al., 1991). Therefore, the 7 aerodynamics of the flow field and related aeroelastic responses of this cylinder are highly 8 unsteady and complicated, attracting a number of studies including the BARC study (Bruno 9 et al., 2014).

Email addresses: dinh.nguyen1@nottingham.ac.uk (Dinh Tung Nguyen), david.hargreaves@nottingham.ac.uk (David M. Hargreaves), john.owen@notingham.ac.uk (John S. Owen) 
For the rectangular cylinder with a long after-body length (if the width-to-depth ratio $B / D \geq 4)$, these shear layers can be trapped underneath circulating flow which is called the separation bubble. The separation bubble can become detached and develops into the leading-edge vortex propagating downstream; its arrival at the trailing edge is phase-locked into the shedding of the trailing-edge vortex and the creation of another leading-edge vortex (Mills et al., 2003). However, this synchronisation is poor and intermittent in the case of the 5:1 rectangular cylinder, where the aforementioned shear layers reattach at points very close to the trailing edge. Wind tunnel experiments found the Strouhal number in this case is not unique; it randomly switches between two values, indicating two different flow regimes (Ozono et al., 1992).

Other literature reveals the effect of the turbulence on the separation bubble. The turbulent wind amplifies the suction peak on the surface and shifts it upstream yielding a smaller separation bubble and earlier pressure recovery (Lee, 1975). Further studies pointed out the turbulence-induced effects on the pressure distribution around a 5:1 rectangular cylinder, including a decrease in the pressure correlation and coherence (Matsumoto et al., 2003).

The elastically supported rectangular cylinder has been found to be prone to the VIV due to the motion-induced vortex shed from the leading edge or the von Kármán vortex shed from the trailing edge (Matsumoto et al., 2008). For a range of aspect ratios from 2.6 to 8, which includes the 5:1 rectangular cylinder, these mechanisms are indistinguishable. In addition, different harmonics of the VIV can be observed, which are associated with different numbers of vortices present along the surface of the body because of the long after-body length.

Further studies on the buffeting response of a bluff body have shown a significant effect of the turbulence on the pressure distribution and aeroelastic behaviour. Matsumoto et al. (1993) reported the turbulence-induced stabilisation effect on the VIV of the rectangular cylinder, due to an increase in the vorticity diffusion and thus a decrease in the strength of vortices. However, Wu and Kareem (2012), Kareem and Wu (2013) and Cao (2015) have recently pointed out the deficiencies in the quantitative and qualitative understanding of the turbulence-induced effect on the VIV of the bluff body with a generic aerodynamic cross section and a bridge deck cross section; studies on the latter were comparatively less than those on the former. A number of collective studies on the circular cylinder reviewed by Cao (2015) showed that the turbulence produces a very strong effect on the VIV lock-in and, in some cases, the turbulence can completely suppress the VIV. Meanwhile, the wind tunnel study conducted by Goswami et al. (1993) suggested that the variation of the VIV structural response of a freely-vibrating circular cylinder in turbulent flow was minimal, compared to that measured in smooth flow. As for bridge deck cross sections including the rectangular cylinder, Kobayashi et al. (1990), Kobayashi et al. (1992), Kawatani et al. (1993) and Kawatani et al. (1999) conducted a series of wind tunnel tests investigating the VIV of rectangular and hexagonal cylinders having different aspect ratios in smooth and turbulent flow. The turbulence suppression effect was not observed for all cross sections. Later, Wu and Kareem (2012) and Kareem and Wu (2013) also pointed out this issue and suggested this was due to the difference in the mechanism of the VIV - whether it was motionedinduced-vortex or von-Kármán-vortex driven VIV. Given that the turbulence does not affect 
the motion-induced vortex, the VIV response can be increased since the turbulence weakens the von Kármán vortex and its mitigation effects on the motion-induced leading-edge vortex (Matsumoto et al., 2008; Wu and Kareem, 2012). Nevertheless, more studies are required to clarify these inconsistencies and provide a more comprehensive explanation on the mechanism of the turbulence-induced effect on the motion-induced vortex and the VIV.

Together with traditional wind tunnel tests, the development of Computational Fluid Dynamics (CFD) allows researchers to model and investigate the aerodynamics of the flow around and the aeroelasticity of the rectangular cylinder. Due to the complexity of the problem and the limitation of computational power, simulations were initially restricted to model the flow around 2D cylinders using Unsteady Reynolds-Averaged Navier-Stokes (URANS) models. Their outcomes agreed well with wind tunnel tests and offered comprehensive explanation of the vortex shedding phenomenon of the rectangular cylinder (Ohya et al., 1992; Tan et al., 1998; Larsen and Walther, 1998). Also, 2D simulations have shown their potential in modelling wind-induced responses and extracting aerodynamic and aeroelastic parameters such as flutter derivatives (Xiang and Ge, 2002; Owen et al., 2006; Sun et al., 2009; Waterson and Baker, 2010). Later, 3D simulations using Large Eddy Simulation (LES) models have become more available, focusing on uncovering the characteristics of the separation bubble, the effect of the after-body length on the separation and reattachment of the flow and the coherence structure of the surface pressure around a static rectangular cylinder (Bruno et al., 2010). LES simulations have also been coupled with structural solvers to model the fluidstructure interaction (FSI) of a 3D elastically supported rectangular cylinder and bridge deck section (Sun et al., 2008; Bai et al., 2013; Zhu and Chen, 2013). These researches highlighted the suitability of the LES model to capture the inherent unsteadiness in FSI problems and to maintain the flow structure in the wake region in contrast to the over-dissipation of the URANS models. Daniels et al. (2014) also applied this method to predict the effect of the freestream turbulence on the aerodynamics of a static and elastically mounted 4:1 rectangular cylinder. The use of the LES model is still limited due to its computationally expensive nearwall treatment and high mesh density. In addition, Bruno et al. (2011) showed that results of 3D LES simulations in the application of the bridge aeroelasticity were very susceptible to the level of discretisation in the span-wise direction. Nevertheless, at the current rate of the computational development, LES simulation is becoming more favourable to investigate the flow field and understand the underlying physical mechanism, such as the aforementioned BARC study.

Extended CFD and wind tunnel studies have been carried out by the authors with the aim to bring more insight into the physical mechanism (i) of the VIV as well as (i) of the turbulence-induced effect on the VIV of the 5:1 rectangular cylinder. The former is the priority focus of this paper. LES simulations and wind tunnel tests are used to investigate the flow field around a sectional model in smooth flow. These two approaches were validated by comparing selective results of static simulations and wind tunnel static tests with the BARC summary statistics. The VIV response of an elastically supported 5:1 rectangular cylinder restrained to the heaving or pitching mode only will be measured in the wind tunnel; these wind tunnel dynamic tests are complemented by corresponding LES simulations. The analysis of the surface pressure distribution with the aid of the Proper Orthogonal Decomposition (POD) reveals the mechanism of the VIV of this particular geometry and associated flow 
97 features. The outcomes in this paper will be further analysed to offer comprehensive expla98 nation of the turbulence-induced effect on the VIV, which will be presented in a separate paper.

100

101

102

103

104

105

\section{CFD Methodology}

The computations were conducted using the open-source CFD software OpenFOAM v2.2.2. The unsteady flow around the rectangular cylinder was modelled using a LES model where the Navier-Stokes equations are spatially filtered by the cell size. With the use of Boussinesq's assumption to express the sub-grid scale tensor, the time-dependent filtered Navier-Stokes equations are written as

$$
\begin{aligned}
\frac{\partial \bar{u}_{i}}{\partial t} & =0 \\
\frac{\partial}{\partial t} \rho \bar{u}_{i}+\frac{\partial}{\partial x_{j}} \rho \bar{u}_{i} \bar{u}_{j} & =-\frac{\bar{p}}{\partial x_{j}}+\frac{\partial}{\partial x_{j}}\left(\mu \frac{\partial \bar{u}_{i}}{\partial x_{j}}\right)+\frac{\partial}{\partial x_{j}}\left[\mu_{S G S}\left(\frac{\partial \bar{u}_{i}}{\partial x_{j}}+\frac{\partial \bar{u}_{j}}{\partial x_{i}}\right)\right]
\end{aligned}
$$

106 where $i$ and $j$ are the tensor notation denoting components of the velocity $u$; $\rho$ and $\mu$ are 107 the fluid density and dynamic viscosity respectively; $\bar{p}$ and $\bar{u}$ are the filtered pressure and 108 velocity. $\mu_{S G S}$ is the sub-grid scale (SGS) viscosity and it is modelled by the use of the 109 conventional Smagorinsky SGS model. To avoid the overestimation of the Smagorinsky 110 constant and to account for the effects of convection, diffusion, production and destruction 111 on the SGS velocity scale, an additional transportation equation is embedded to determine 112 the distribution of the kinetic energy of the SGS eddies, $k_{S G S}$, and the SGS viscosity , $\mu_{S G S}$, 113 (Furby et al., 1997)

$$
\begin{aligned}
& \frac{\partial}{\partial t} \rho k_{S G S}+\frac{\partial}{\partial x_{j}} \rho k_{S G S} \bar{u}_{j}=\frac{\partial}{\partial x_{j}}\left(\mu_{S G S} \frac{\partial k_{S G S}}{\partial x_{j}}\right)+2 \mu_{S G S} \bar{S}_{i j} \bar{S}_{i j}-C_{\varepsilon} \frac{k_{S G S}^{3 / 2}}{\Delta}, \\
& \mu_{S G S}=\rho C_{S G S} \Delta k_{S G S}^{1 / 2},
\end{aligned}
$$

114 where the constants are $C_{\varepsilon}=1.048$ and $C_{S G S}=0.094 . \Delta$ is the characteristic length scale of 115 the filter, which is defined as the cubic root of the cell volume. To remove the over-dissipation 116 of the kinetic energy in the near-wall region, a filtered width $\delta$ according to the van Driest 117 approach is introduced as

$$
\begin{aligned}
\delta & =\min \left\{\Delta, \frac{k}{C_{\Delta}} y\left(1-\exp ^{-\frac{y^{+}}{A^{+}}}\right)\right\}, \\
y^{+} & =\frac{\sqrt{\rho \tau_{w}}}{\mu} y
\end{aligned}
$$


118 where $k=0.4187$ is the von Kármán constant, $C_{\Delta}=0.158$ and $A^{+}=26$ are the van 119 Driest constants. $\tau_{w}$ is the wall shear stress and $y$ and $y^{+}$are the normal distance and 120 non-dimensional normal distance to the wall respectively.

121 The domain geometry and some key boundary conditions are summarised in Figure 1. 122 For the purposes of this computational study, the width of the cylinder. $B=0.5 \mathrm{~m}$, and the 123 depth $D=0.1 \mathrm{~m}$. The span-wise length of the cylinder and the width $L$ of the domain was $1243 B$. The blockage ratio was $4.8 \%$. A zero gradient condition for velocity and a constant value 125 of zero gauge pressure were imposed on the outlet. As for the inlet, a non-zero $x$-component 126 wind speed and a zero gradient condition for pressure were specified to simulate smooth flow. 127 The OpenFOAM boundary condition movingWallVelocity was applied on the surface of 128 the model to accurately model a zero normal-to-wall velocity component at a moving wall. 129 The symmetryPlane boundary condition was used for the two $z$ patches while the cyclic 130 boundary condition was selected for the two $y$ patches.

131 The meshing operation to the domain geometry was conducted using ANSYS-Meshing 132 within Workbench and OpenFOAM utilities, fluentMeshToFoam and extrudeMesh. As a 133 result, the computational domain was discretised using a 3D hybrid hexahedral grid, where 134 the grid was hybrid in the $x-z$ plane (Figure 2a) and structured in the $y$ direction. An 135 inflation layer which was a six-cell-thick structured grid was imposed around the model where 136 the thickness of cells next to the model was $\Delta z / B=2 \times 10^{-3}$ and grew by a ratio of 1.2 137 (Figure $2 \mathrm{~b}$ ). The discretisation in the along-wind direction was constant $\Delta x / B=2 \Delta z / B$. 138 The unstructured hexahedral grid was used for the remaining part of the $x-z$ plane. The 3D 139 grid was constructed by projecting the 2D grid along the $y$ direction; 30 layers was used with 140 the span-wise discretisation $\Delta y / B=0.1$, resulting in a total of 2.1 million cells (Figure 3 ).

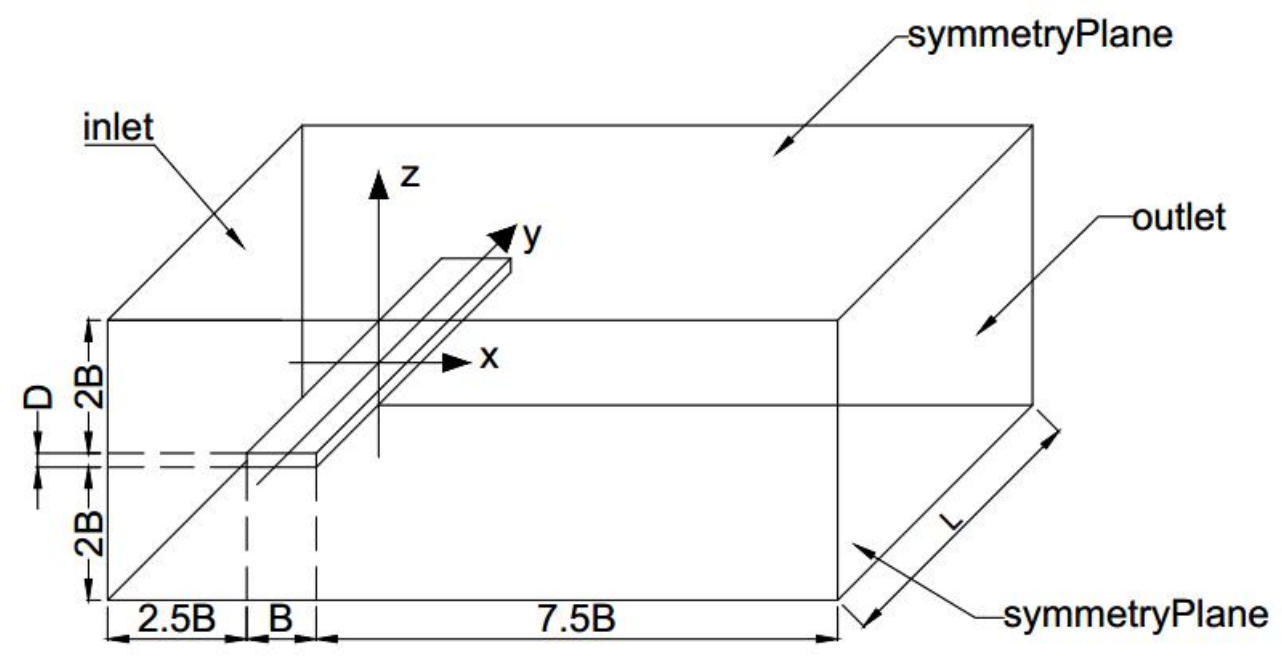

Figure 1: Domain geometry and boundary conditions of selected patches. 
(a)

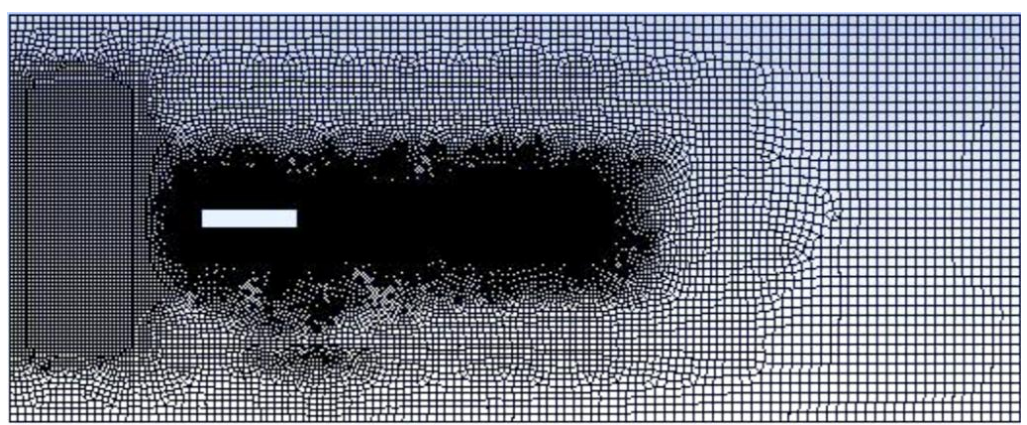

(b)

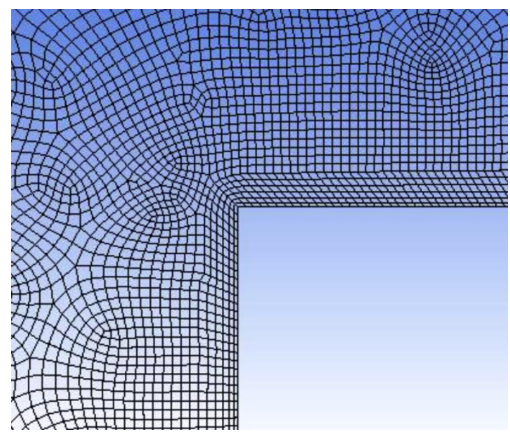

Figure 2: The computational grid in the $x$ - $z$ plane (a) for the entire domain and (b) around the leading edge.

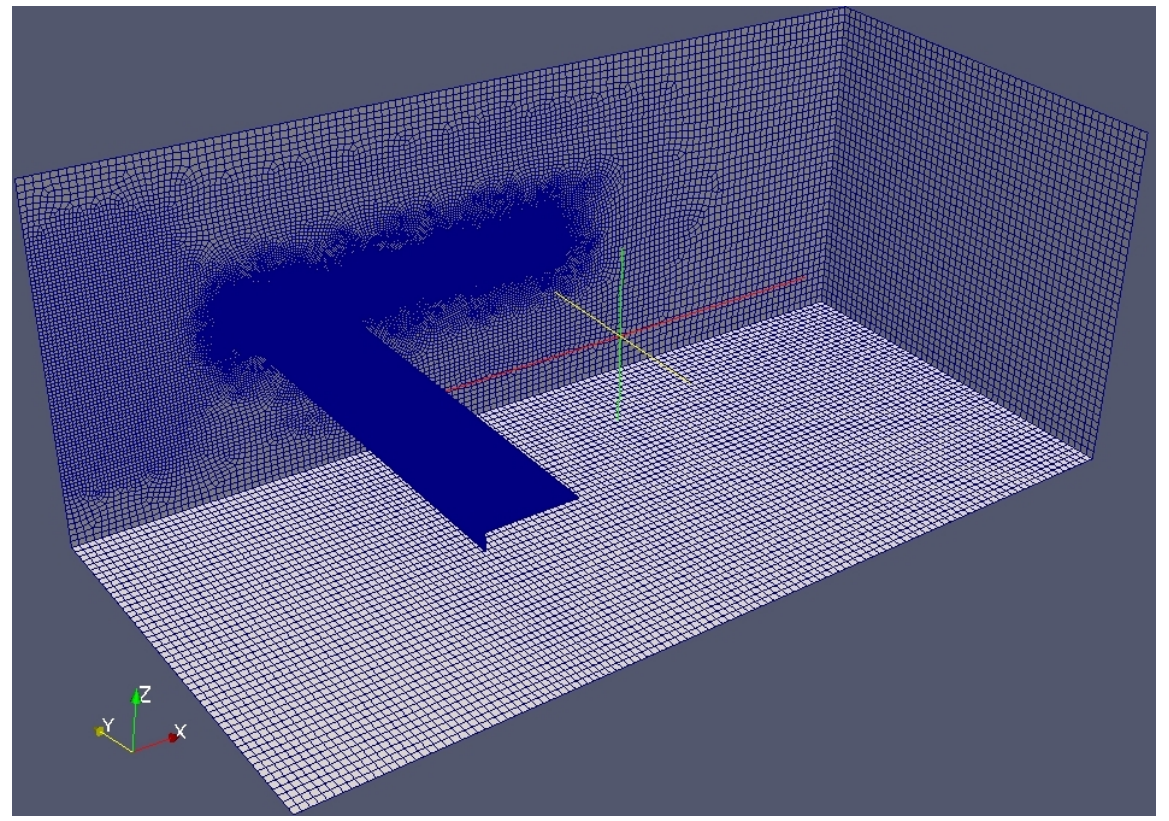

Figure 3: The computational grid used in the 3D heaving simulation.

141 The pressure-velocity coupling was achieved by means of the PIMPLE algorithm, which 142 is a merged PISO-SIMPLE solver. During one time-step, two PISO loops were performed, 143 leading to better coupling between pressure and velocity and allowing bigger time-steps and, 144 hence, Courant number. The governing equation was spatially discretised using second145 order schemes. The convection terms were discretised by the use of the limited linear 146 scheme while the second-order central differencing scheme was applied to the diffusion terms. 147 Non-orthogonal correction factors were enabled to take into account the skewness and non148 orthogonality of the unstructured grid. As for the temporal discretisation, the implicit 149 second-order backward difference scheme was selected. The non-dimensional time-step $\Delta t^{\star}=$ $150 \Delta t U / B\left(\Delta t\right.$ is the time-step and $U$ is the upstream mean wind speed) was $2 \times 10^{-3}$. 


\subsection{Static Simulations}

The OpenFOAM solver pimpleFoam was used to simulate the flow around the 3D static sectional model at 3 different wind speeds, 1, 2 and $4 \mathrm{~m} \mathrm{~s}^{-1}$. At each wind speed, the static force and moment coefficients were measured and the surface pressure was extracted at selected points to evaluate the pressure distribution and span-wise correlation. Each simulation was extended over 80 non-dimensional time $t^{\star}=t U / B$ to obtain converged statistics and data in further 120 non-dimensional time was used to perform analysis. Static simulations were conducted in parallel on the High Performance Computer (HPC) at the University of Nottingham. One simulation was computed in parallel using 32 processors and 8 GB of memory; it would take from 1 to 1.5 months to produce reliable data for analysis.

\subsection{Dynamic Simulations}

The coupling procedure between the OpenFOAM solver pimpleDyMFoam and the massdamp-spring equation was utilised to perform the dynamic simulation. The model was restrained to respond in the heaving mode only with the natural frequency of $f_{n, h}=1.2 \mathrm{~Hz}$. The model has a mass per unit length of $\bar{m}=4.7 \mathrm{~kg} \mathrm{~m}^{-1}$; the damping ratio was $\zeta_{h}=1 \%$ yielding the Scruton number $\operatorname{Scr}=\left(\pi \bar{m} \zeta_{h}\right) /(\rho B D)$ of 8.97. The wind speed was increased from $0.1 \mathrm{~m} \mathrm{~s}^{-1}$ to $2.5 \mathrm{~m} \mathrm{~s}^{-1}$ in increments of $0.1 \mathrm{~m} \mathrm{~s}^{-1}$ during the lock-in interval.

A new dynamic mesh class Foam: :dynamicHeavingFreeUDFFvMesh was written and implemented in OpenFOAM to model the heaving motion of the model; this dynamic class is also capable to model the pitching response. It contains a structural solver where the mass-damping-spring equation was discretised and solved using the the first-order backward Euler method

$$
\begin{aligned}
\ddot{z}_{n+1} & =\frac{F_{n}}{\bar{m} L}-2 \omega_{n, h} \zeta_{h} \dot{z}_{n}+\omega_{n, h}^{2} z_{n}, \\
\dot{z}_{n+1} & =\dot{z}_{n}+\Delta t \ddot{z}_{n+1} \\
z_{n+1} & =z_{n}+\Delta t \dot{z}_{n+1}
\end{aligned}
$$

173 where $z_{i}, \dot{z}_{i}, \ddot{z}_{i}$ and $F_{i}$ are the displacement, velocity and acceleration of and the force acting 174 on the model at the time step $i ; \Delta t$ is the time-step size. The model has the angular natural 175 frequency $\omega_{n, h}$ and the damping ratio $\zeta_{h}$. Here, the backward Euler method was selected 176 since it can better model the implicit nature of the FSI problem.

A dynamic mesh algorithm was implemented based on the linear-spring-analogy algorithm proposed by Batina (1990). Since the maximum displacement during the lock-in is relatively epth of the cross section) and the computational domain was sinple, this dynamic mesh algorithm is a plausible solution, still maintaining good cell qualities. The

181 computational domain was divided into 9 blocks (Figure 4). Blocks 8 and 9 are rigid where 182 all grid nodes are fixed relative to the model. The other blocks are grouped into a buffer 183 zone where cells are allowed to deform to facilitate the displacement of the model. The 184 conventional serial staggered algorithm was applied to model the coupling between the fluid, 185 structure and dynamic mesh. 
186 Each dynamic simulation was computed in parallel on the HPC using 32 processors and 18710 GB of memory. The physical time for the dynamic simulation was similar to that applied 188 in the static simulation; this was sufficient for the transient period to settle down and the 189 fluid and structure solutions to reach the stable oscillatory state. One dynamic simulation 190 at one wind speed took from 1 to 1.5 months to finish.

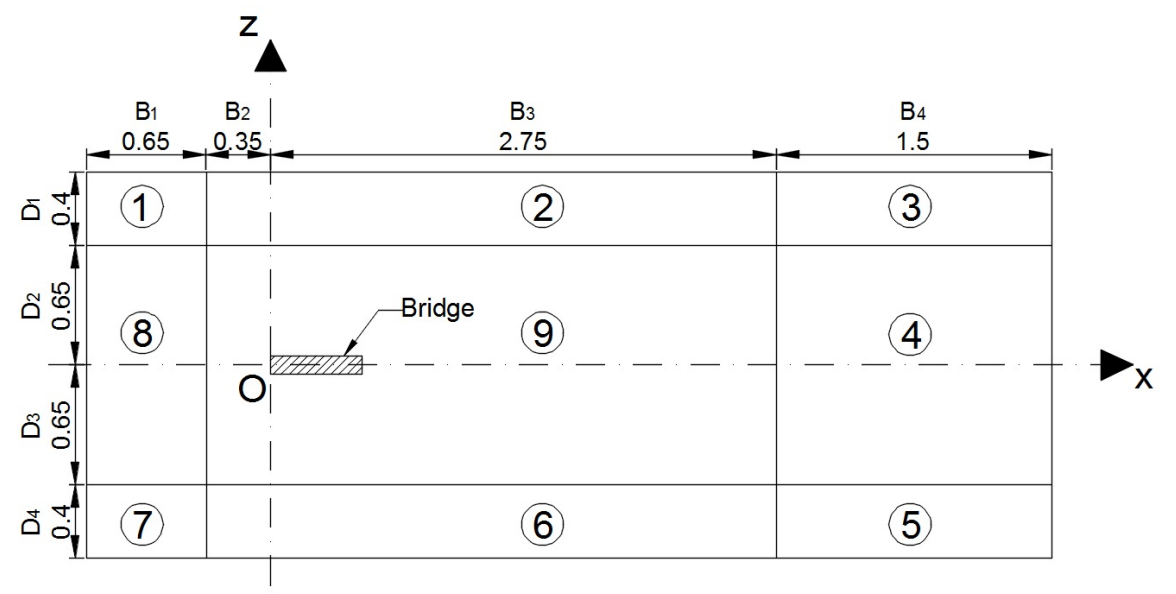

Figure 4: Illustration of 9 different blocks in the computational domain; dimensions are in metres.

\section{3. Wind Tunnel Methodology}

192 The wind tunnel tests were conducted at the Atmospheric Boundary Layer wind tunnel at 193 the University of Nottingham. For aerodynamic tests such as the work described here, the low 194 turbulence section immediately downwind of the contraction was used. With no additional 195 turbulence generation, there is a uniform flow away from the walls, with a turbulence intensity 196 of less than $0.2 \%$. Both static and dynamic tests were conducted in smooth flow.

197 The $5: 1$ rectangular model is $1.6 \mathrm{~m}$ long with a $0.308 \mathrm{~m}$ by $0.076 \mathrm{~m}$ section; these dimen198 sions result in a blockage ratio of $2.89 \%$. The model was instrumented by 7 arrays of pressure 199 taps as shown in Figure 5a. There are 16 pressure taps distributed around the cross section 200 at each array as shown in Figure 5b. Each tap was connected to an individual pressure sensor 201 HCLA02X5DB from First Sensor. 
(a)

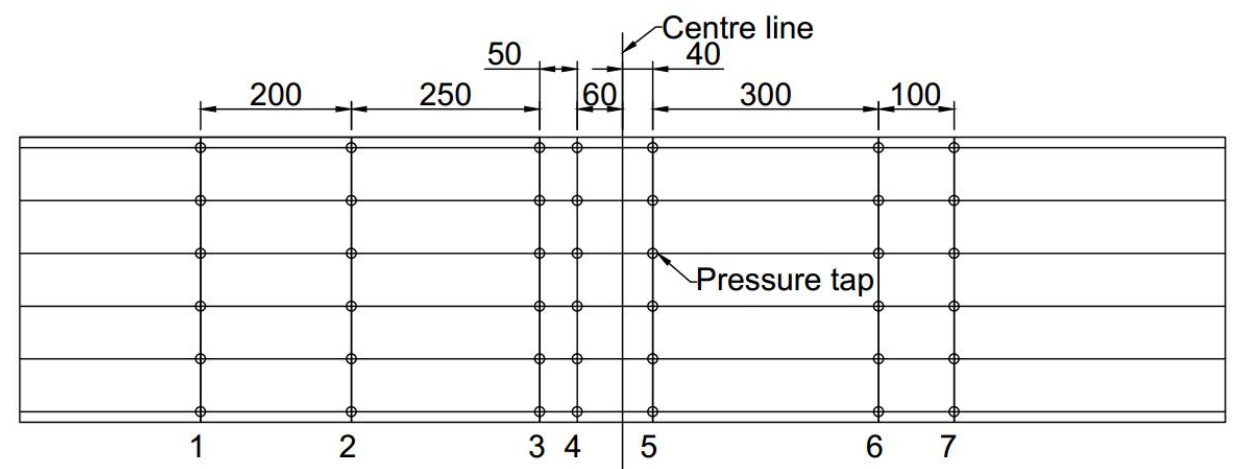

(b)

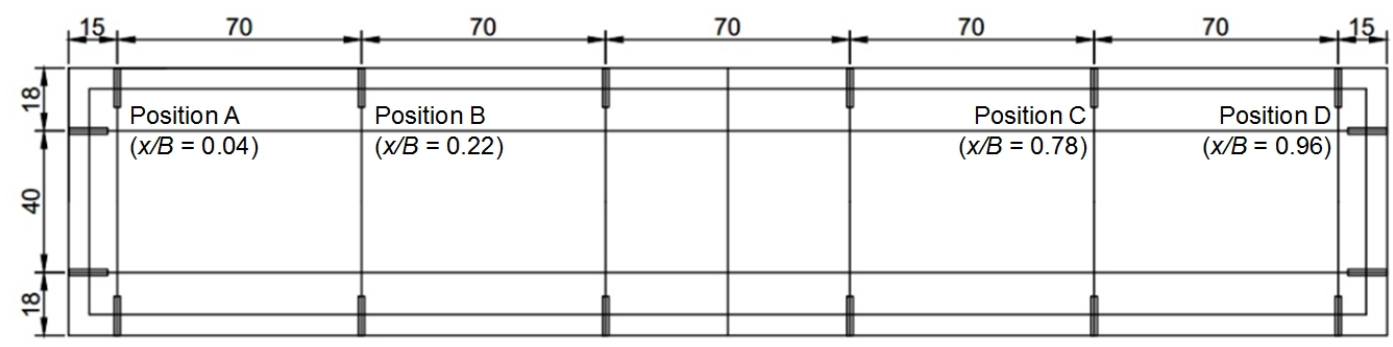

Figure 5: (a) Arrangement of pressure taps on the bottom surface and (b) a cross section of the model showing the distribution of pressure taps at each array; dimensions are in $\mathrm{mm}$ ).

\subsection{Static Test Procedures}

For the static tests, the model was rigidly supported on load cells in a frame within the aerodynamic working section. These load cells comprised of six compression force sensors (Kistler 9313AA1) and were manufactured at the University of Nottingham. The model was tested for 4 different wind speeds $4,6,8$ and $10 \mathrm{~m} \mathrm{~s}^{-1}$ and at the angle of attack $0^{\circ}$. A X-wire probe (TSI 1241-T1.5) was placed at a distance, $B$, behind the trailing edge and a distance, $D$, above the top surface to investigate the flow structure in the wake. At each wind speed, the surface pressure was measured and force coefficients were calculated from the load cell data.

\subsection{Dynamic Test Procedures}

The dynamic test was set up as shown in Figure 6; the sectional model was mounted on eight E0750115500S springs supplied by Associated Spring Raymond and restrained by light wires so that it responded in the heaving or pitching mode only. The natural frequency and damping ratio of the heaving were measured to be $f_{n, h}=4.68 \mathrm{~Hz}$ and $\zeta_{h}=0.19 \%$ respectively $(\mathrm{Scr}=15.9)$. For the pitching mode, the natural frequency and damping ratio were $f_{n, p}=5.70 \mathrm{~Hz}$ and $\zeta_{p}=0.13 \%$ respectively. The wind speed was increased from 1 to $10 \mathrm{~m} \mathrm{~s}^{-1}$. A coarse step size of $0.5 \mathrm{~m} \mathrm{~s}^{-1}$ was used outside the lock in region; whereas during the lock-in, a small increment of $0.1 \mathrm{~m} \mathrm{~s}^{-1}$ was applied to accurately track changes in dynamic behaviour. At each wind speed, the response was recorded using 2-axis MEMS 
221 accelerometers ADXL203 by Analog Devices, mounted on four corners of the model, and 222 the surface pressure was measured. A TSI X-wire 1241-T1.5 probe was located at the same 223 position as was used in the static tests to capture the the wake velocity. In all static and 224 dynamic wind tunnel tests, the acceleration, forces and pressure were sampled at $500 \mathrm{~Hz}$ 225 while the velocity in the wake was sampled at $1000 \mathrm{~Hz}$.

226 It should be noticed that, for the work presented here, selective results of the static 227 simulation and wind tunnel static tests were compared with the BARC summary statistics 228 to validate the computational and wind tunnel approaches.

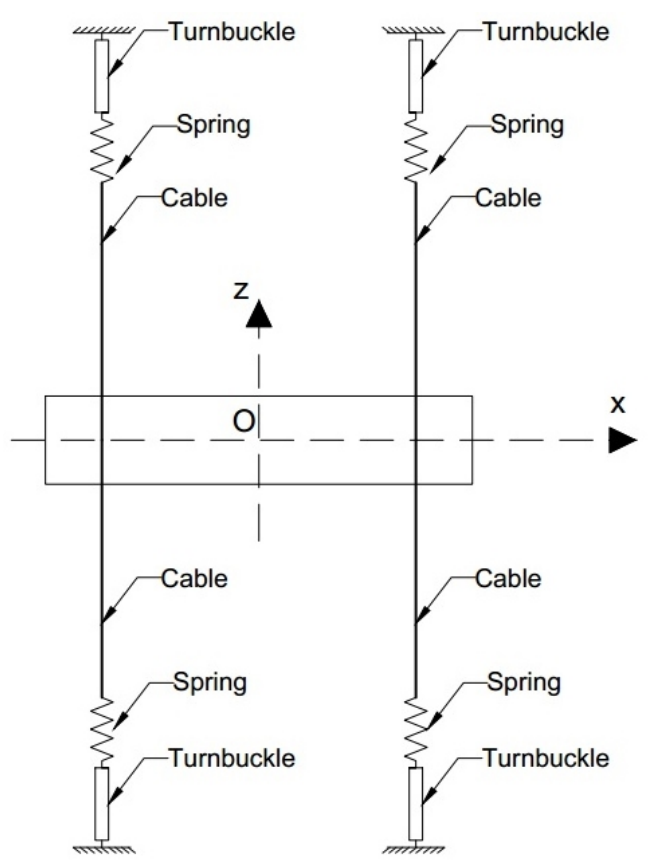

Figure 6: Schematic of the set-up of the dynamic test. 


\section{Validation Study}

\subsection{Mesh Sensitivity Study}

To evaluate the effect of the span-wise discretisation on the flow field being modelled by LES, a mesh sensitivity study was conducted by performing the static simulation at the wind speed of $1 \mathrm{~m} \mathrm{~s}^{-1}$ using four grids in Table 1. They have different span-wise discretisation levels $\Delta y / B$ and similar grids on the $x-z$ plane. Grid G4 is the computational domain used in the dynamic simulation. The Strouhal number, St, was extracted from the power spectral density of the lift force and was plotted against the quantity $(\Delta x \Delta y \Delta z)^{1 / 3} / B$ (Figure 7 ). This scaling factor was selected to be representative of the domain since it is the filtering width applied to solve fluid solutions in the region next to the model and reflects the spanwise discretisation. The Grid Convergence Index (GCI) was then calculated to estimate uncertainties regarding the spatial discretisation of the domain (Roache, 1997; Celik et al., 2008). As a result, the numerical uncertainty of the dynamic simulation was $\mathrm{GCI}_{\text {fine }}^{34}=28 \%$; the Strouhal number predicted by the grid G4 was within the BARC summary statistic of wind tunnel and computational results (Bruno et al., 2014). However, as shown in Figure 7 , the independence of the Strouhal number from the mesh was not achieved; the use of finer span-wise discretisation leads to a higher Strouhal number.

Figure 8 also indicates some influence of the span-wise discretisation on the pressure distribution. For the time-averaged pressure coefficient $C_{p}$ (Figure 8a), all four profiles stayed within the BARC envelops. The pressure fluctuation inside the separation bubble modelled in the four simulations was in good agreement with the BARC statistics. However, the pressure recovery region showed more scatter (Figure $8 \mathrm{~b}$ ). The overall trend was that a coarser grid predicted higher pressure fluctuation; results from Grids 3 and 4 were about $5 \%$ to $30 \%$ larger than the upper envelop of the BARC statistics.

Therefore, it is evident that the flow field around the rectangular cylinder was significantly affected by the span-wise discretisation. By reducing the discretisation level in this direction, i.e. using a larger filtering width, certain small-scale flow features would not be resolved properly. This affected the energy flow and energy dissipation of large-scale vortices, which eventually influenced the Strouhal number and the surface pressure distribution. These observations were agreed by arguments of Celik et al. (2005) that the mesh convergence of LES is impossible to achieve. Both numerical errors associated when resolving most-energetic eddies and SGS errors when modelling SGS eddies depend on the filtering width or, in this case, the cell size. A decrease in the cell size gradually reduces these errors; eventually, the mesh convergence is achieved when the cell size becomes so small that LES simulation is equivalent to Direct Numerical Simulation. In addition, there is a limitation on this mesh sensitivity study that only cells in the span-wise direction were refined, while cells in the $x-z$ plane remained unchanged. This means that the refinement produced more positive effects on the fluid solutions on the $y$ direction more than those on the $x$ and $z$ directions. This issue was more pronounced in case of high aspect-ratio cells such as those used in this computational study. 
Table 1: Computational grids in the mesh sensitivity study.

\begin{tabular}{ccc}
\hline Grid & $\Delta y / B$ & Number of layers \\
\hline G1 & 0.01 & 300 \\
G2 & 0.02 & 150 \\
G3 & 0.04 & 75 \\
G4 & 0.1 & 30 \\
\hline
\end{tabular}

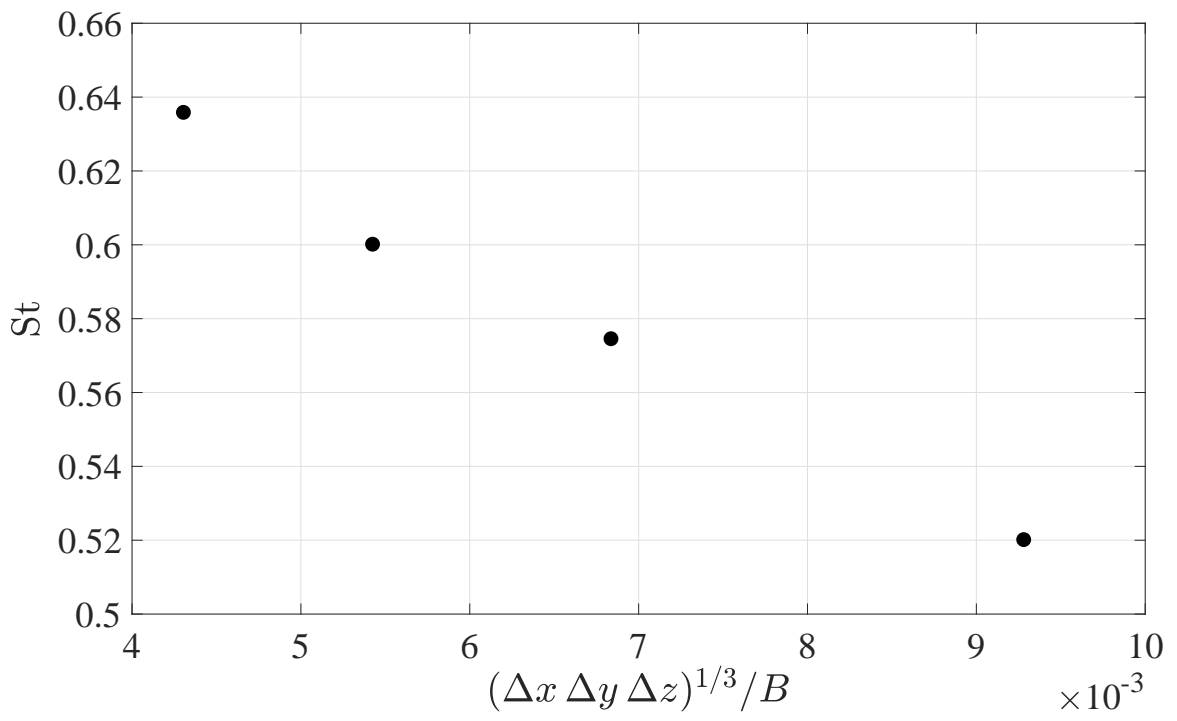

Figure 7: Variability of the Strouhal number, St, against the quantity $(\Delta x \Delta y \Delta z)^{1 / 3} / B$, which is the normalised filtering width applied to solve fluid solutions in the region next to the model. 
(a)

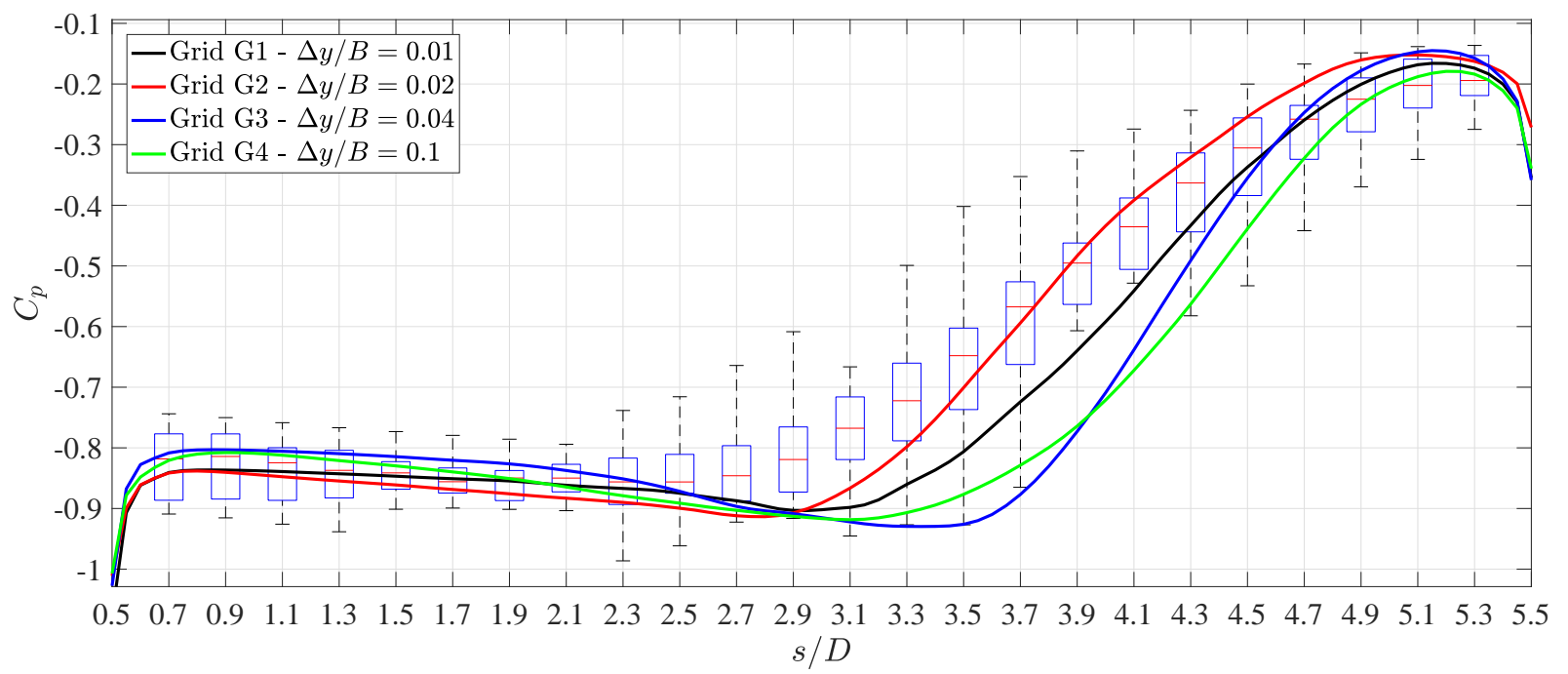

(b)

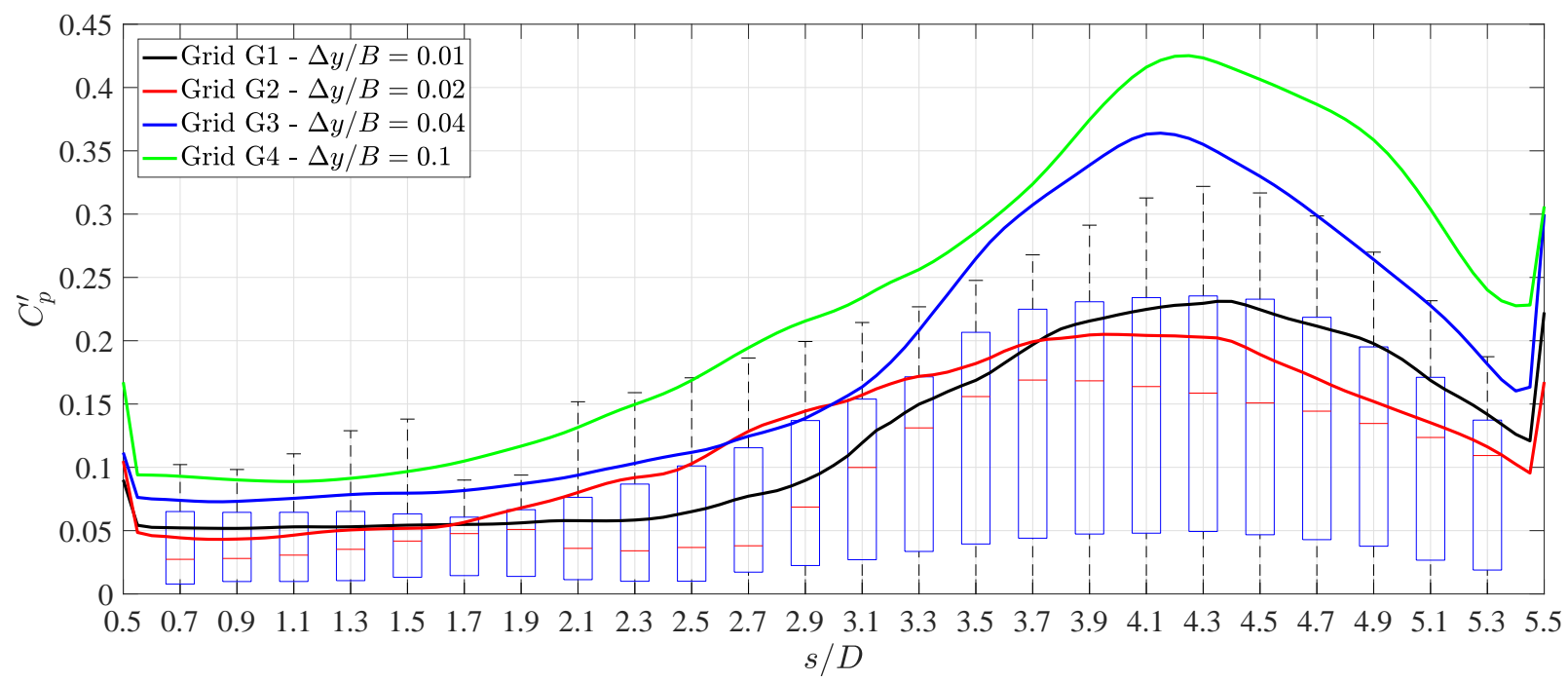

Figure 8: The surface distribution of (a) the time-averaged pressure coefficient $C_{p}$ and (b) the standard deviation of the time-varying pressure coefficient $C_{p}^{\prime}$ in comparison to the BARC summary statistics of CFD simulations (Bruno et al., 2014).

\subsection{Comparison with BARC Summary Statistics}

To further validate the computational and wind tunnel approaches, the force coefficient, the Strouhal number and the surface pressure distribution around the cylinder at the angle 272 of attack $0^{\circ}$ were compared against the BARC summary statistics. As shown in Table 2, the 273 time-averaged drag coefficient, $C_{D}$, the standard deviation of the time-varying lift coefficient, $274 C_{L}^{\prime}$, and the Strouhal number, St, are in a good agreement with selected BARC studies (Bruno 275 et al., 2010; Schewe, 2013). Also, results of the computational study are within the BARC 276 summary statistics of computational results (Bruno et al., 2014). The time-averaged lift 
Table 2: Comparison of force coefficients and Strouhal number obtained from computational and wind tunnel studies; $(\star)$ numbers in the brackets are the standard deviation of computational results reported in BARC.

\begin{tabular}{llcccc}
\hline & Re & St & $C_{D}$ & $C_{L}$ & $C_{L}^{\prime}$ \\
\hline \multirow{3}{*}{ CFD study } & 6700 & 0.608 & 0.241 & -0.056 & 0.081 \\
& 13000 & 0.600 & 0.206 & -0.059 & 0.075 \\
& 27000 & 0.609 & 0.206 & -0.063 & 0.059 \\
\hline \multirow{2}{*}{ WT study } & 20800 & 0.640 & 0.225 & -0.0811 & 0.0784 \\
& 31200 & 0.621 & 0.230 & -0.0684 & 0.0848 \\
& 41600 & 0.622 & 0.240 & -0.0690 & 0.0932 \\
& 52000 & 0.601 & 0.252 & -0.0706 & 0.115 \\
\hline WT study & $6000-40000$ & 0.555 & 0.242 & $\sim 0$ & $\sim 0.08$ \\
\hline $\begin{array}{l}\text { CFDewe, 2013) } \\
\text { (Bruno et al., 2010) }\end{array}$ & 40000 & 0.575 & 0.206 & - & $\sim 0.146$ \\
\hline $\begin{array}{l}\text { BARC statistics of CFD } \\
\text { (Bruno et al., 2014) }\end{array}$ & - & 0.545 & 0.2148 & -0.00282 & 0.130 \\
\hline
\end{tabular}

277

coefficient, $C_{L}$, however displays the largest deviation from the BARC data. Issues regarding the accurate setting up angles of attack and correcting the blockage ratio were the major contribution to errors in the wind tunnel study. For the computational study, the negative $C_{L}$ indicated an asymmetric flow field around the cylinder. This could be attributed to the use of the unstructured grid where the cell density and cell size were slightly different between the top and bottom halves of the domain.

Another useful measure of the quality of the experimental measurements and numerical predictions are the surface pressure correlation measured along the leading and trailing edges and the surface pressure distribution. In Figure 9, despite different Reynolds numbers, the pressure correlation obtained from the static simulations and wind tunnel static tests show similar trends and reasonably agree with the BARC summary statistics and a selected wind tunnel test of Ricciardelli and Marra (2008). The use of the cyclic boundary condition on the $y$ patches is thought to increase the pressure correlation beyond $\Delta y / B=0.7$ to 0.8 ; this issue was also observed by Mannini et al. (2011). In general, the pressure correlation measured along the leading edge is higher than that measured along the trailing edge. These results indicated the presence of the separation bubble which was well-defined along the span-wise direction in comparison to a highly unsteady flow feature close to the trailing edge.

These two flow features can also be inferred from the surface pressure distribution obtained from the wind tunnel static tests (Figure 10). The separation bubble close to the leading edge was a well correlated recirculating flow feature, which was trapped underneath the shear layer generated from the leading edge. The separation bubble induced strong suction, with little variation, on the surface of the cylinder (from $s / D=0.7$ to 2.5 approximately). Close the trailing edge, this shear layer reattached to the surface, leading to a recovery and large variation of the surface pressure (from $s / D=3.3$ to 4.3 approximately). These flow features 
301 are associated with the impinging vortex shedding phenomenon, which is well documented in the literature (Nakamura et al., 1991; Mills et al., 2003; Bruno et al., 2010, 2014). The timea good agreement between results of the wind tunnel static tests and the BARC summary statistics can be seen.

(a)

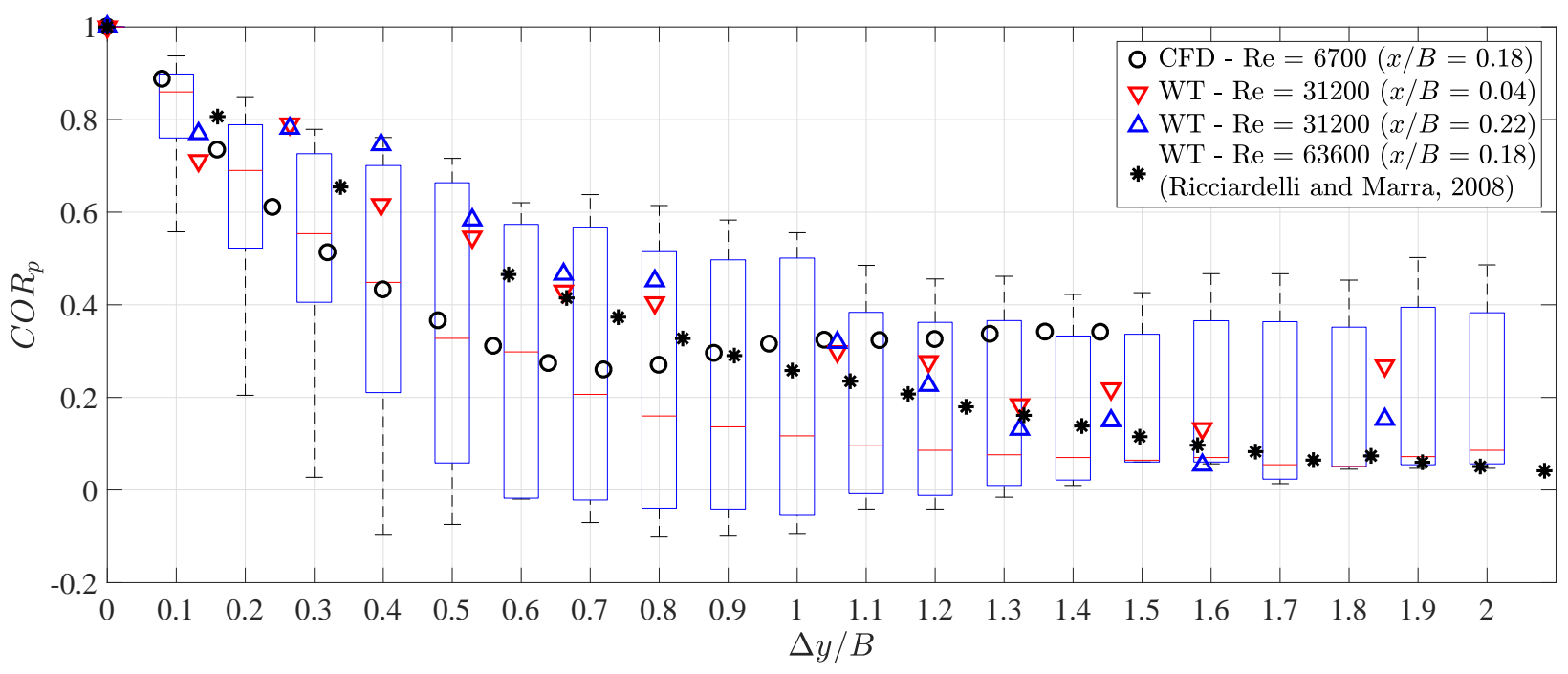

(b)

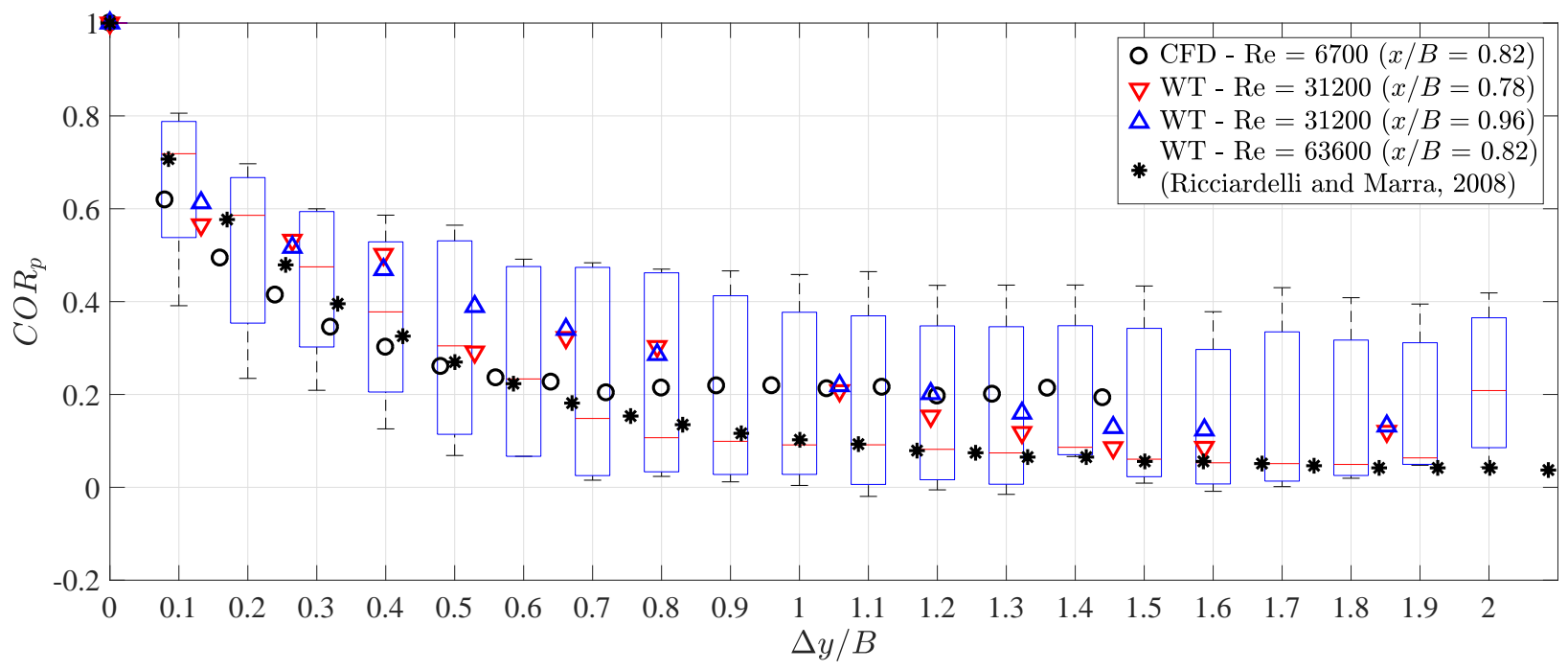

Figure 9: Comparison of the surface pressure correlation along (a) the leading edge and (b) the trailing edge, measured in the CFD static simulations and WT static tests, against a selected wind tunnel test and the BARC summary statistics of CFD simulations and WT tests (Bruno et al., 2014). 
(a)

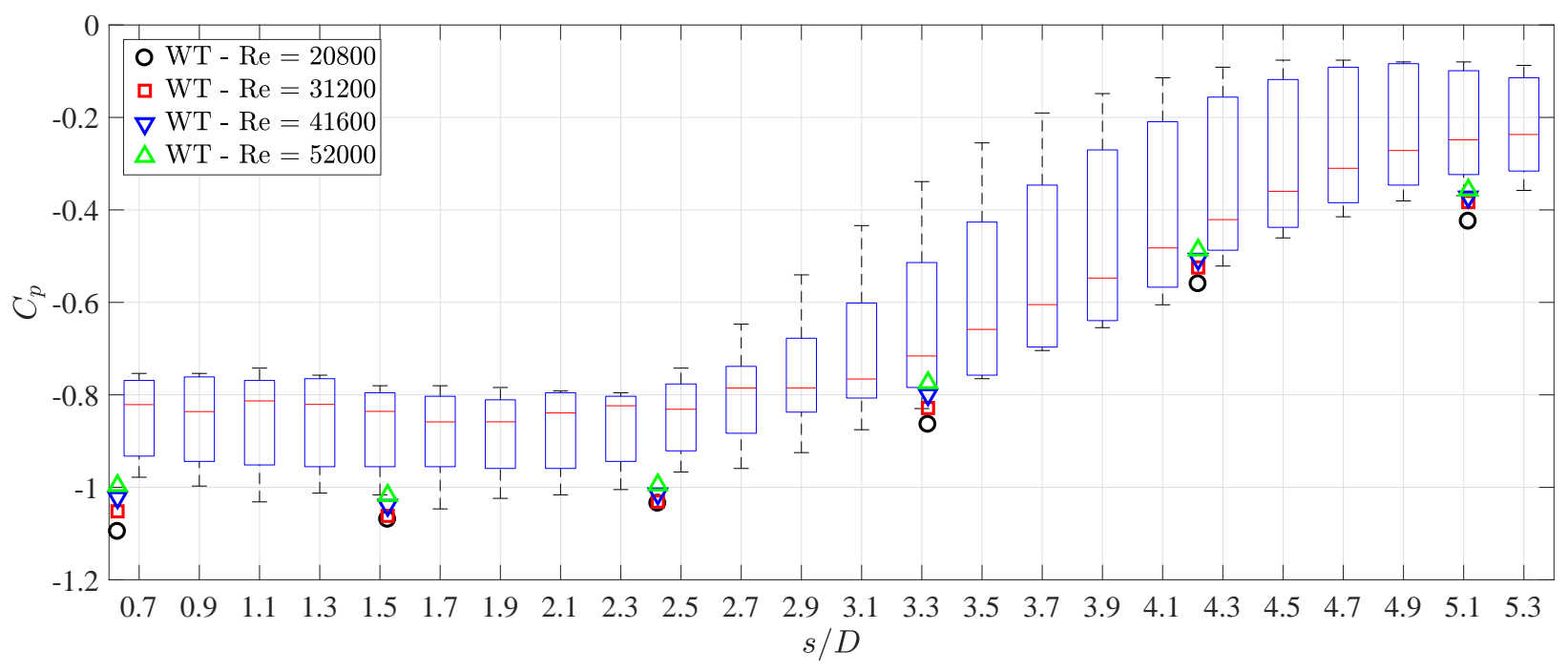

(b)

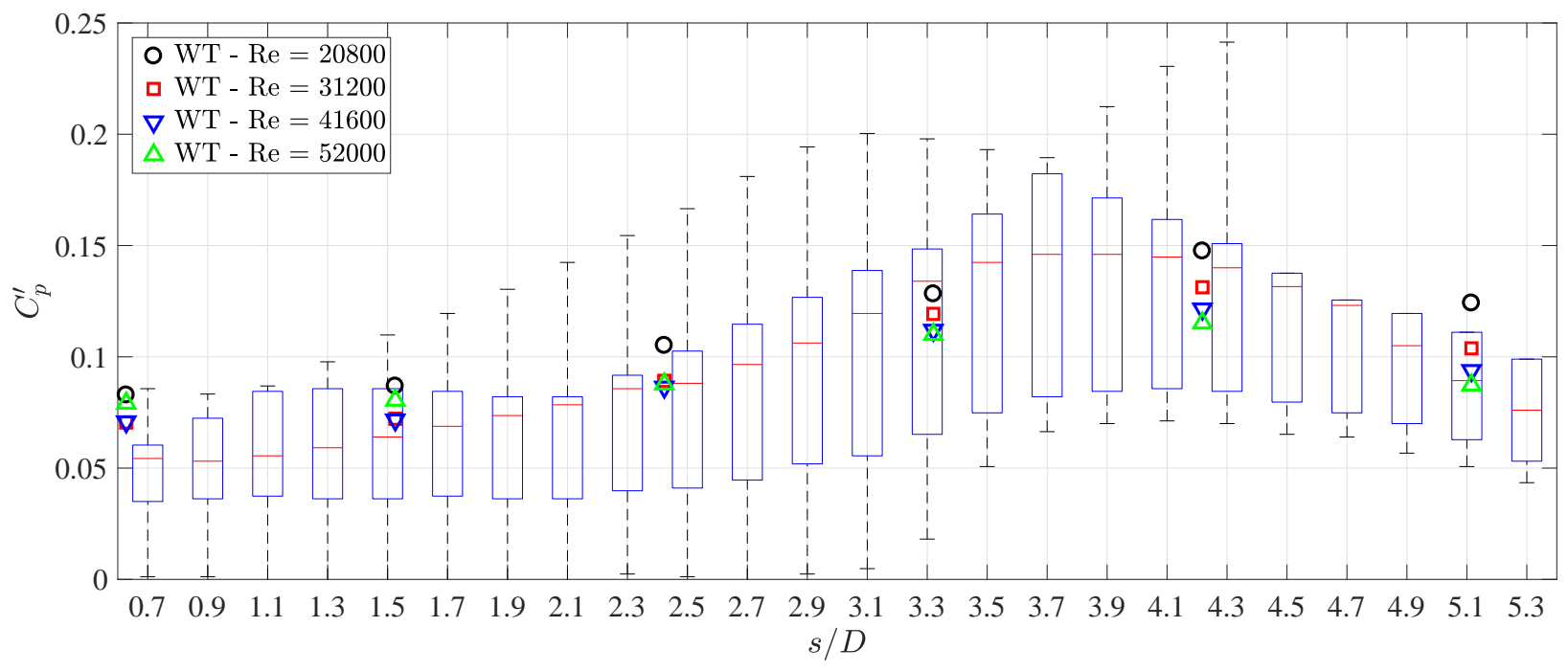

Figure 10: Comparison of the surface distribution of (a) the time-averaged pressure coefficient, $C_{p}$, and (b) the standard deviation of the time-varying pressure coefficient, $C_{p}^{\prime}$, measured in the WT static tests, against the BARC summary statistics of WT tests (Bruno et al., 2014).

By means of a comparison with the BARC summary statistics, the methods applied 307 in this study have been validated, particularly for the numerical modelling regarding the 308 computational domain, numerical schemes and associated effects, which were the dependence 309 of the Strouhal number and flow field on the span-wise discretisation. These methods were 310 then used to carry out the dynamic simulations and wind tunnel dynamic tests. Results and 311 further discussion are presented in the next sections. 


\section{VIV Mechanism of the 5:1 Rectangular Cylinder}

313 The heaving VIV of the rectangular cylinder was both measured in the wind tunnel and 314 modelled computationally while the pitching VIV was measured in the wind tunnel only. 315 A comparison of results obtained from these two studies in smooth flow was conducted 316 to provide a comprehensive explanation of the VIV mechanism. Using this finding, results 317 obtained from the wind tunnel in turbulent flow were then analysed to uncover the mechanism 318 of the turbulence-induced effect on the VIV.

\subsection{Heaving $V I V$}

Figures 11 and 12 summarise results as the rectangular cylinder underwent heaving VIV, measured in the wind tunnel and 3D heaving simulation respectively. Due to the absence of a reliable force measurement during the wind tunnel test, information related to the lift force or moment was retrieved by performing the integration of the surface pressure measured at the pressure array 4 (Figure 5a). The pressure measurement was associated with certain limitation of the sensitivity at low wind speeds; therefore, results of force and moment were limited in the this range of wind speeds. Also, the presence of the rolling motion impaired results of the vortex shedding frequency and the phase shift of the lift force, which is indicated by some fluctuation in Figures $11 \mathrm{c}$ and $11 \mathrm{~d}$ at the reduced wind speed $U_{R}=U /\left(f_{n, h} B\right)$ of 1.4 and 2.5 respectively.

Results obtained from wind tunnel tests and computational simulations possess similar trends. Both studies predicted two VIV lock-in intervals indicated by an increase in the structural response and the fact the vortex shedding frequency was locked into the natural frequency of the model. Due to the larger Scruton number (higher mass and damping ratio), the wind tunnel test predicted lower structural responses during the VIV lock-in compared to the ones predicted by the computational simulation.

In the wind tunnel dynamic test, two heaving VIV lock-in regions occurred at the onset reduced wind speed $U_{R \text {,onset }}=0.77$ and 1.54; the former was smaller in magnitude (Figure 11a). Similarly, the 3D heaving simulation predicted two VIV lock-in intervals at $U_{R \text {,onset }}=1$ and 2 (Figure 12a). The former peak was smaller in magnitude; as was revealed by the phase analysis of the surface pressure shown in Figure 13, this peak was associated with two vortices alternately being formed on the top or bottom surfaces of the model during one cycle of motion. This contrasted with there being only one vortex on the side when the model experienced the larger response. This difference in the flow structure could also be observed in the instantaneous contour plots of the Q-criterion (Figure 14). The main vortices are enclosed by red squares while the blue square highlights the secondary vortex resulted from the interaction of the main ones. As suggested by Nakamura et al. (1991) and Matsumoto et al. (2008), the number of vortices appearing on one side of the cylinder during one cycle of motion, $n$, is related to the onset reduced wind speed of the VIV heaving lock-in such that $U_{R \text {,onset }}=n /$ St. This relationship allowed the Strouhal number to be estimated; good agreement with results obtained from wind tunnel static tests and static simulation presented in Table 2 could be drawn. 
(a)

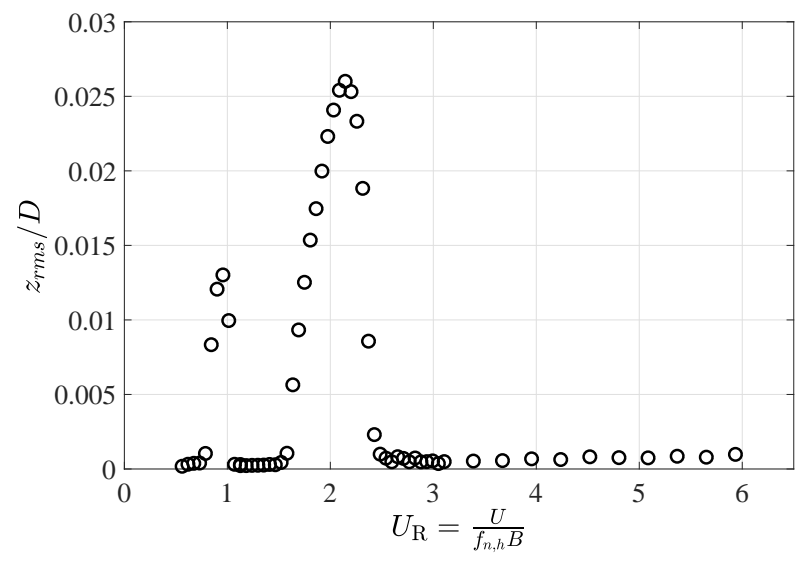

(c)

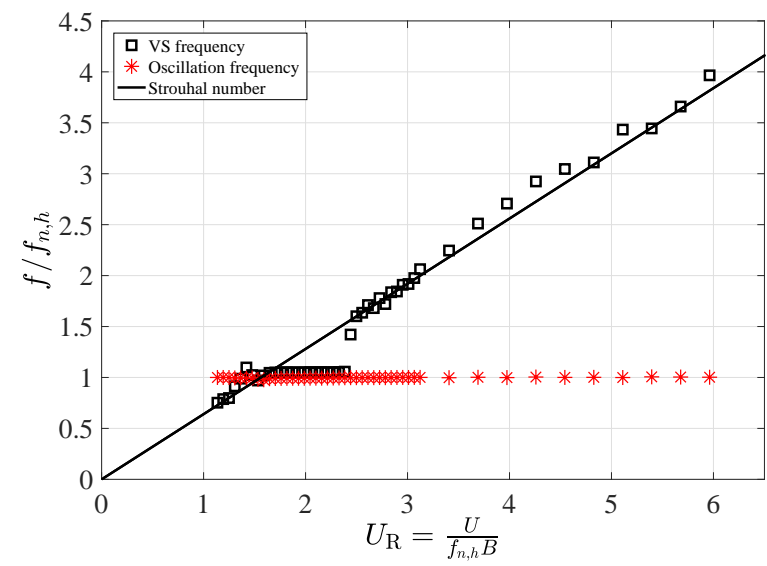

(b)

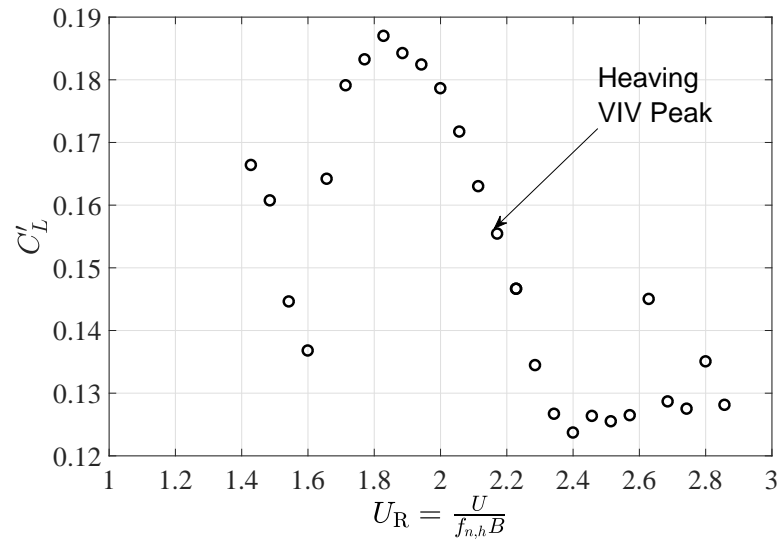

(d)

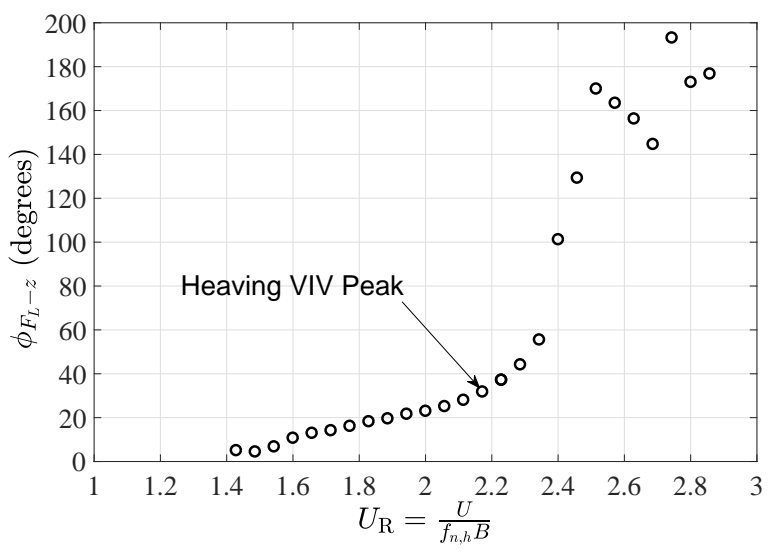

Figure 11: Results of the wind tunnel dynamic test of the sectional model restrained to the heaving mode only: (a) structural response, (b) lift coefficient response, (c) frequency of response and (d) phase shift of the lift force against the structural displacement. 
(a)

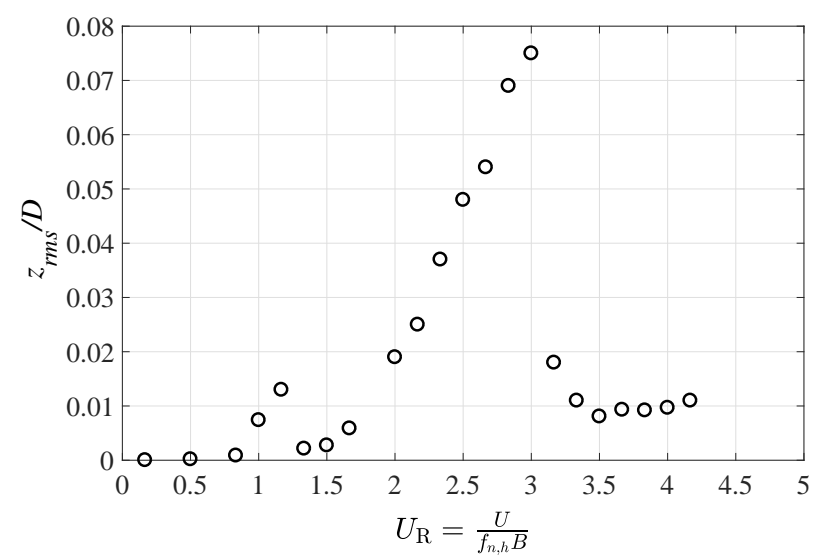

(c)

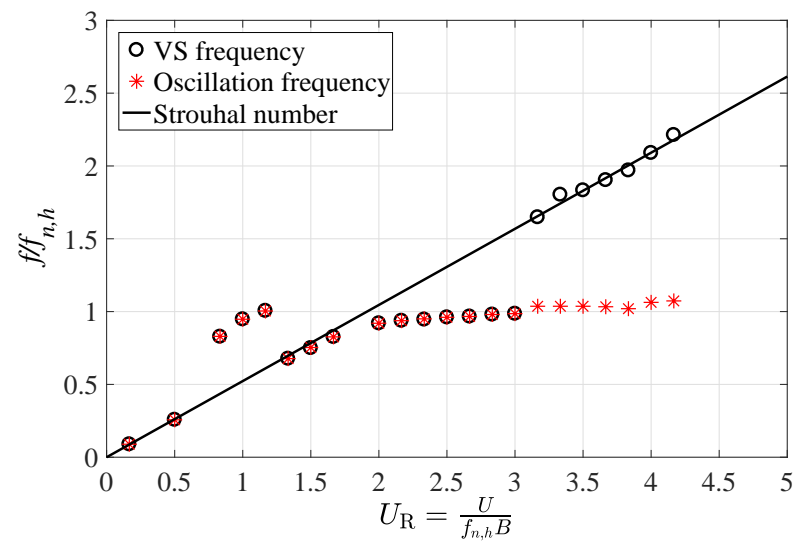

(b)

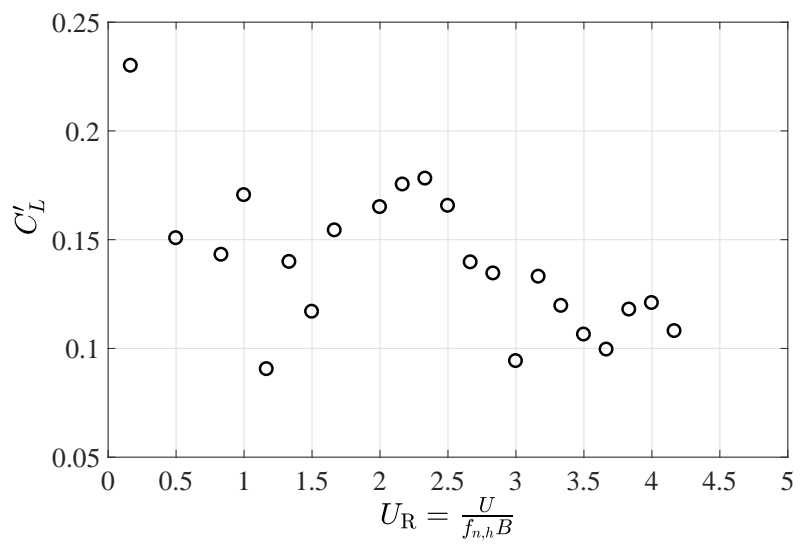

(d)

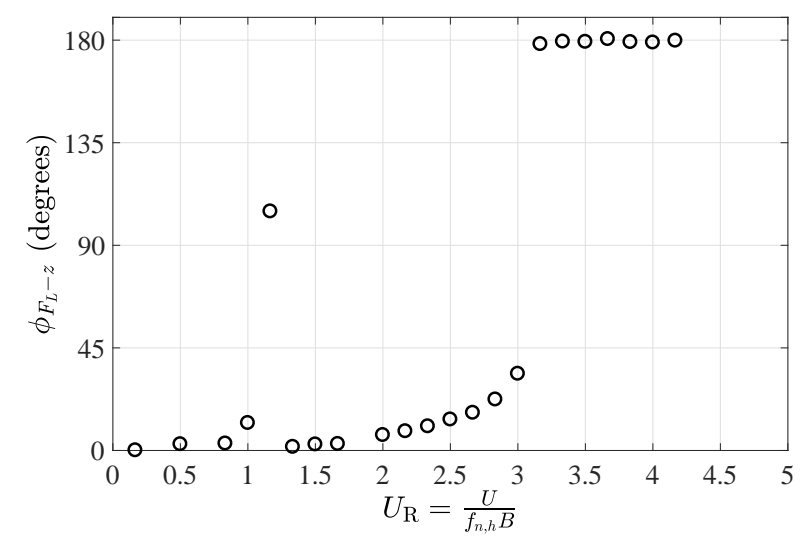

Figure 12: Results of the 3D heaving simulation of the sectional model: (a) structural response, (b) lift coefficient response, (c) frequency of response and (d) phase shift of the lift force against the structural displacement. 
(a) Secondary VIV peak

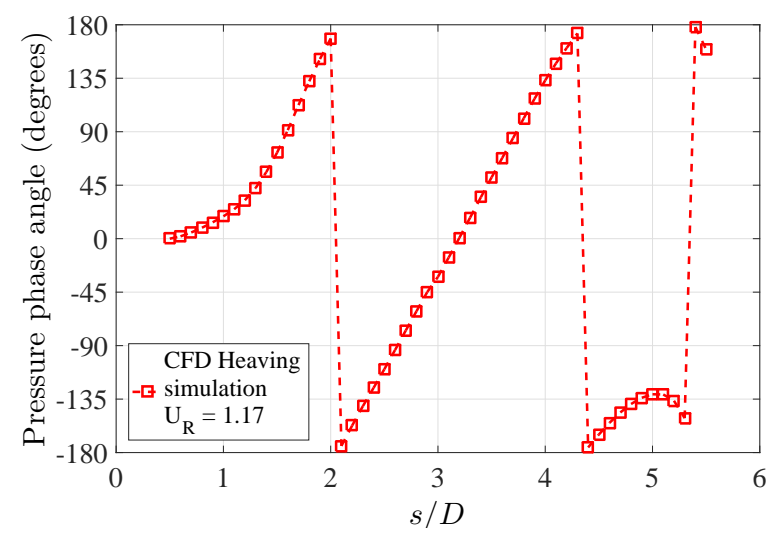

(b) Primary VIV peak

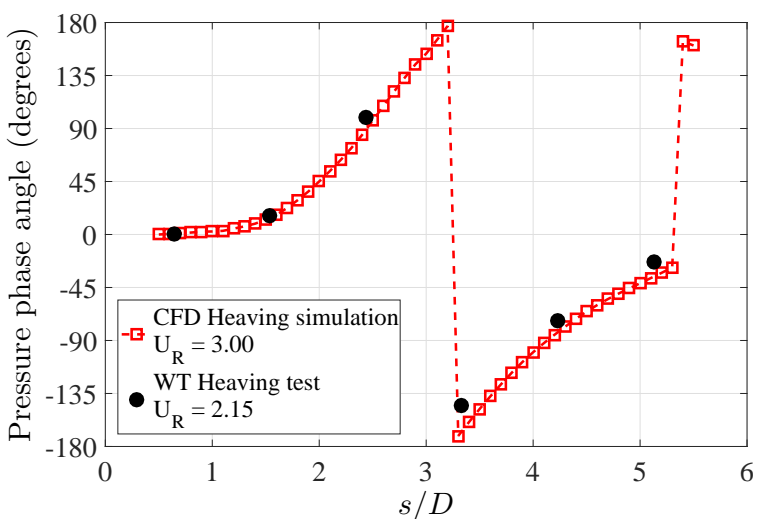

Figure 13: Phase angles of vortices rolling on the surface of the cylinder measured in the wind tunnel dynamic test and in the 3D heaving simulation; all results are calculated at the reduced wind speeds corresponding the maximum structural displacement during the lock-in.

(a)

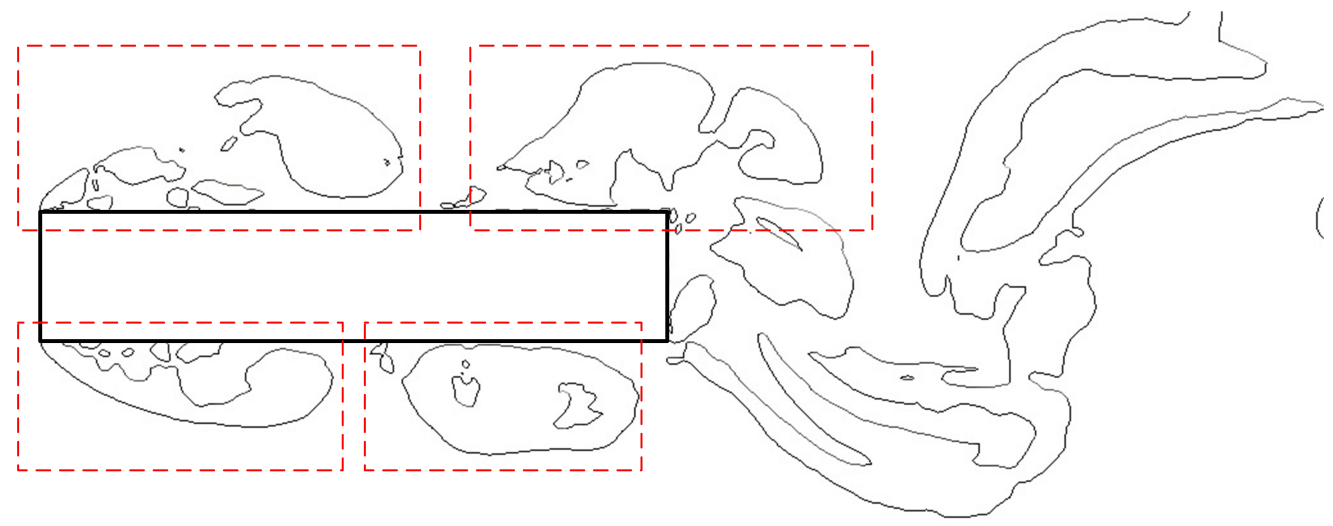

(b)

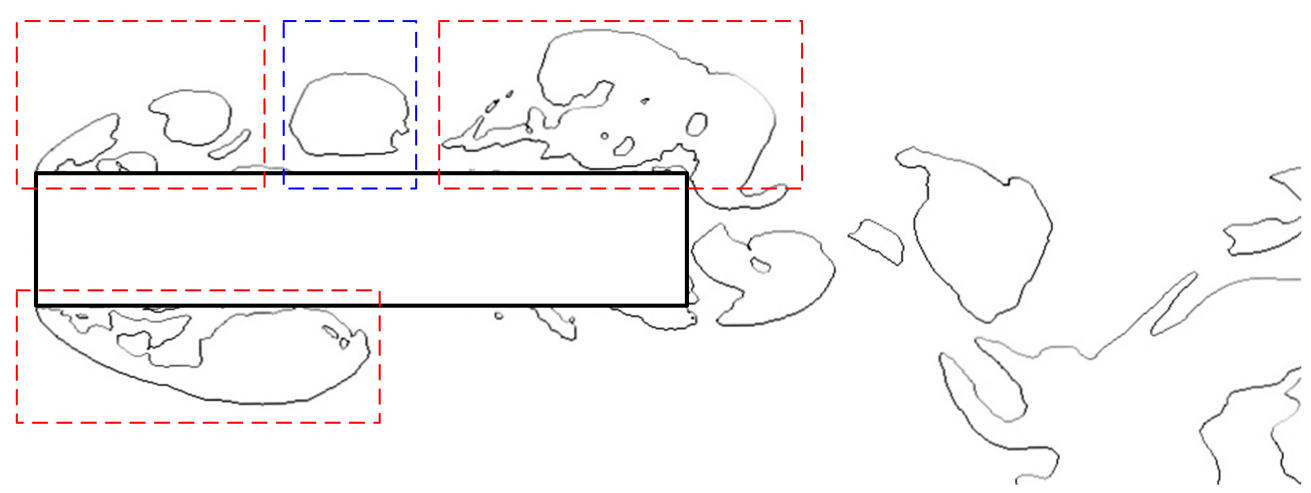

Figure 14: Contour plots of the Q-criterion $Q=0.1 \mathrm{~m} \mathrm{~s}^{-1}$ along the mid-span plane at (a) $U_{R}=1.17$, i.e. the secondary VIV peak and (b) $U_{R}=3.00$, i.e. the primary VIV peak; results were obtained from the 3D heaving simulation. 
Both the wind tunnel test and the 3D heaving simulation predicted similar behaviour for 353 the phase shift of the lift force against the displacement of the cylinder as shown in Figures $35411 \mathrm{~d}$ and $12 \mathrm{~d}$. As the amplitude of the structural response increased, the in-phase component 355 of the lift force became less dominant and after the cylinder reached the lock-out, the lift 356 force suddenly became out-of-phase. This transition also indicated that there was a dramatic 357 change in the flow structure around the cylinder which was responsible for the lock-out; this 358 will be revealed further by analysing the span-wise correlation of the surface pressure as the 359 heaving VIV lock-in occurred.

Concentrating on the primary peak of the heaving VIV measured in the wind tunnel (Positions A and B) and around the traing 362 (Positions A and B) and around the trailing edge (Positions C and D) is illustrated in 363 Figure 15; the locations of these four positions are indicated in Figure 5b. Before the lock364 in occurred, the pressure correlation around the leading edge was higher than that around 365 the trailing edge, illustrating the presence of the leading edge vortex. The increase in the 366 amplitude of the response improved the correlation of the surface pressure. However, during 367 the lock-in, the correlation level around Position $\mathrm{C}$ was higher than those around the leading 368 edge. This result indicated a strongly correlated flow feature occurred at Position C every 369 cycle of the motion and it led to an increase in the response whereas the motion-induced 370 371 leading-edge vortex was only responsible for triggering the motion. It was noticed that the span-wise pressure correlation exhibited an increase at $\Delta y / B=1$. This was caused the rolling motion of the cylinder, coupling with a finite span-wise length of the model and the end plates, which resulted in a standing wave effect superimposed on the flow field.

374

375

376

377

378

379

380

381

382

383

384

385

386

387

388

389

390

391

392

393

394

Results obtained from the heaving simulation also revealed similar behaviour (Figure 16). Before the VIV lock-in $\left(U_{R}=1.67\right)$, the flow field around the leading edge was better correlated than the one around the trailing edge. When the lock-in occurred and the amplitude of the response increased $\left(U_{R}=2.00\right.$ to 2.67$)$ and reached the peak $\left(U_{R}=3.00\right)$, a slight decrease in the correlation level around the leading edge was observed while, around the trailing edge, the flow field was better correlated. When the system reached the lock-out, the correlation level around the trailing edge suddenly decreased.

Figure 17 describes the variation of the pressure field on the top surface at $U_{R}=3.00$ during one cycle of the structural motion $\left(T_{n, h}\right)$ extracted from the computational simulation. The pressure field presented here is the dominant component resulted from a Proper Orthogonal Decomposition analysis. At the start of the cycle of structural motion $t=0$, there was a vortex being shed from the leading edge; the downward motion of the cylinder from $t=0$ to $T_{n, h} / 2$, however, significantly affected its span-wise geometry, degrading its span-wise correlation and causing it to propagate downstream. In the next quarter of the cycle, due to the upward accelerating movement of the cylinder, this motion-induced leading-edge vortex dramatically slowed down and appeared to impinge on the surface of the cylinder. During this process, this vortex gained strength and its span-wise correlation improved; this increased the lift force acting on the cylinder in the direction such that the cylinder was effectively brought back to the equilibrium position. In the final quarter of the cycle, thanks to the decelerating upward motion of the cylinder, this vortex was pushed downstream at a higher rate and was eventually shed into the wake. The behaviour of the motion-induced leading- 
395 edge vortex during one cycle of the heaving motion is summarised in Figure 18. Together the 396 wind tunnel dynamic test, these results from the computational simulation indicated that, 397 particularly for the 5:1 rectangular cylinder, the motion-induced leading-edge vortex acted 398 as a triggering mechanism for the VIV response while the impingement of this vortex on 399 the surface of the cylinder close to the trailing edge resulted in an increase in the structural 400 response during the lock-in.

(a) $U_{R}=1.76$

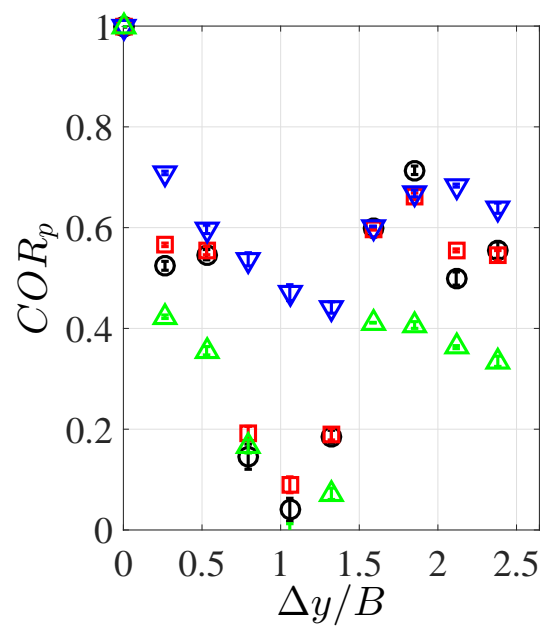

(b) $U_{R}=1.82$

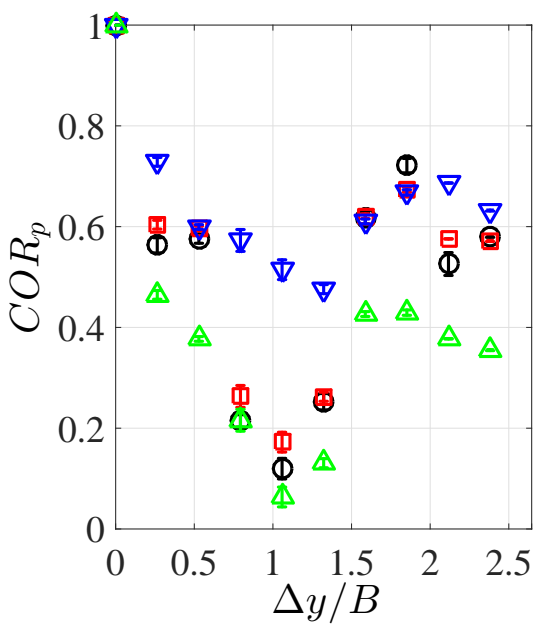

(c) $U_{R}=2.16$

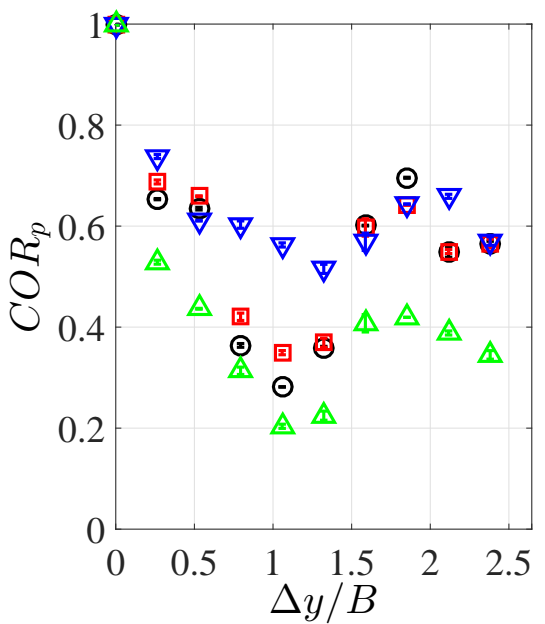

Figure 15: Wind tunnel results of the span-wise pressure correlation measured at 4 stream-wise positions in the smooth flow during the heaving VIV lock-in; black: Position A; red: Position B; blue: Position C; green: Position D.

(a) Leading edge $(x / B=0.18)$

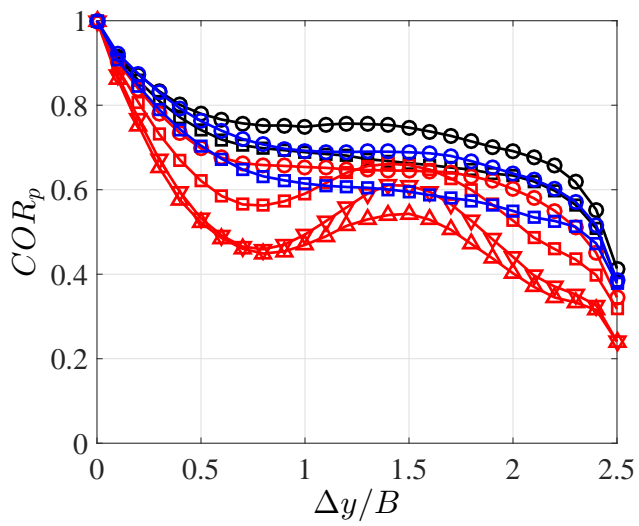

(b) Trailing edge $(x / B=0.82)$

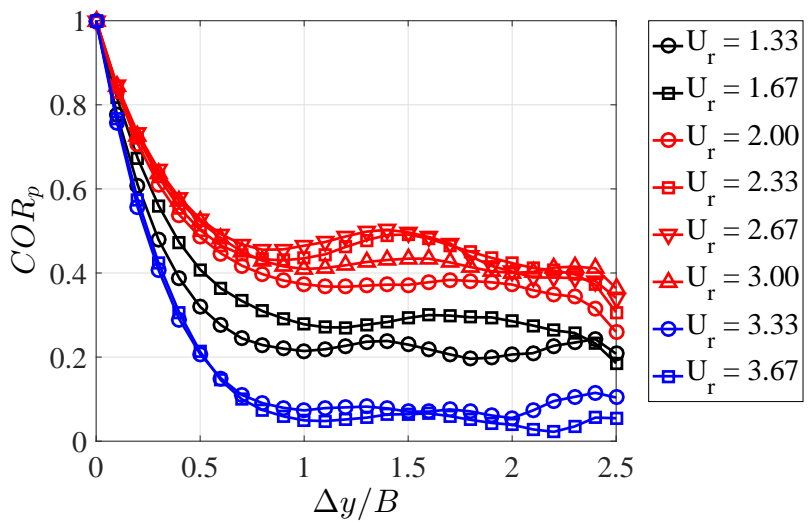

Figure 16: Computational results of variation of the span-wise pressure correlation around the leading and trailing edges as the cylinder experienced the heaving VIV lock-in; black: before the lock-in; red: VIV lock-in; blue: after the lock-in. 
(a) $t=0$
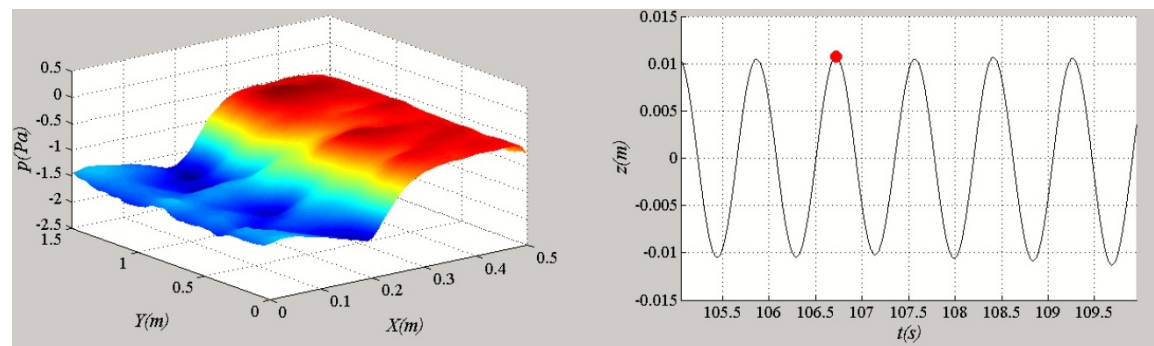

(b) $t=T_{n, h} / 4$
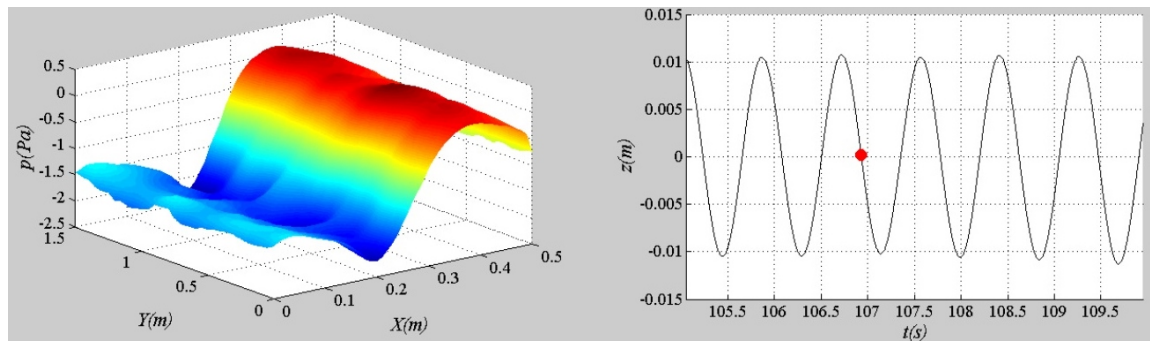

(c) $t=T_{n, h} / 2$
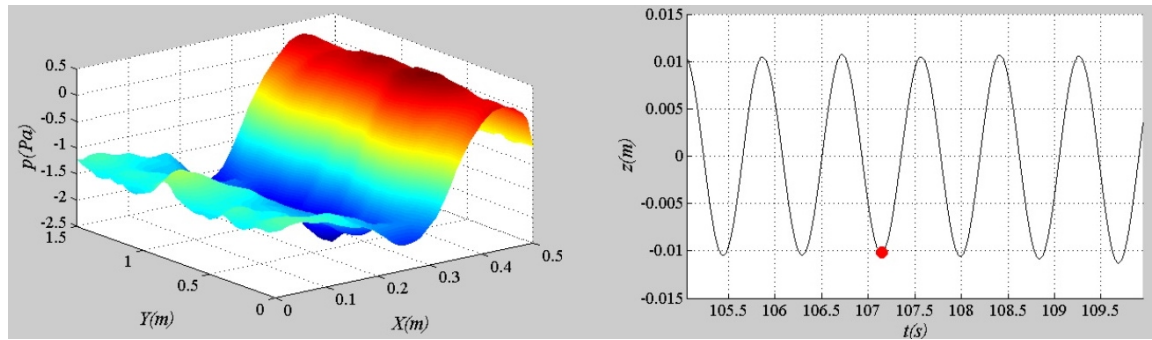

(d) $t=3 T_{n, h} / 4$
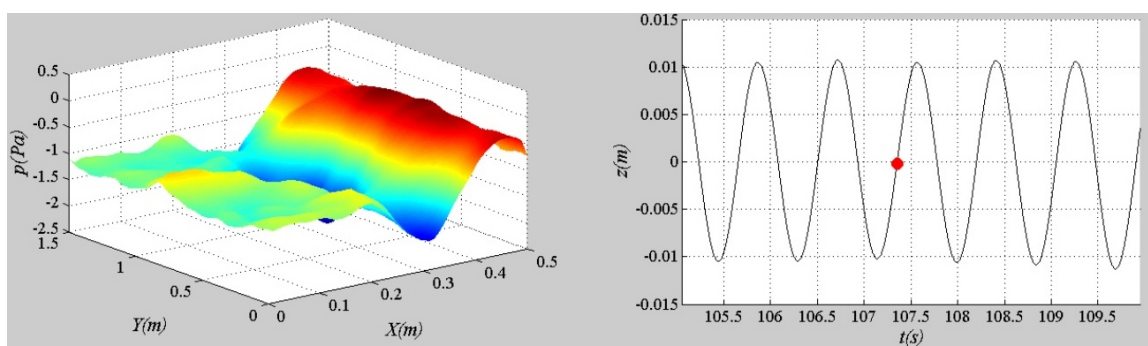

(e) $t=T_{n, h}$
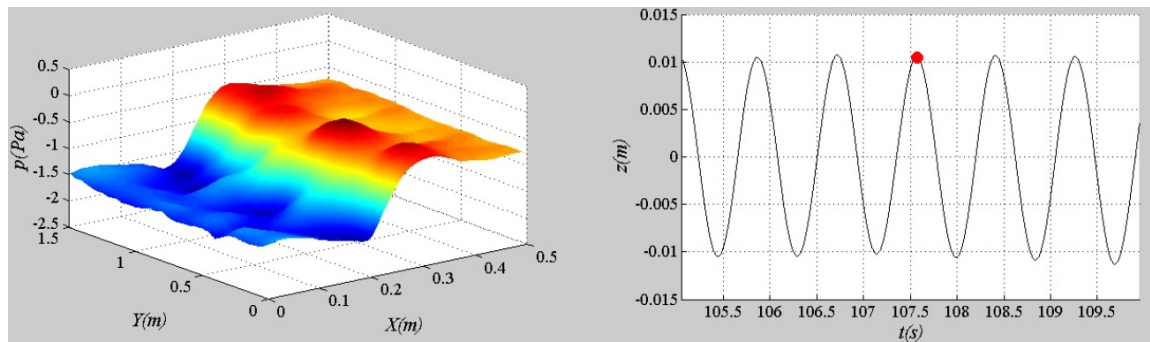

Figure 17: The pressure on the top surface of the cylinder at every quarter of the cycle of the structural oscillation $\left(T_{n, h}\right)$ obtained from the computational simulation; the red dot indicates the position of the cylinder during the cycle. 


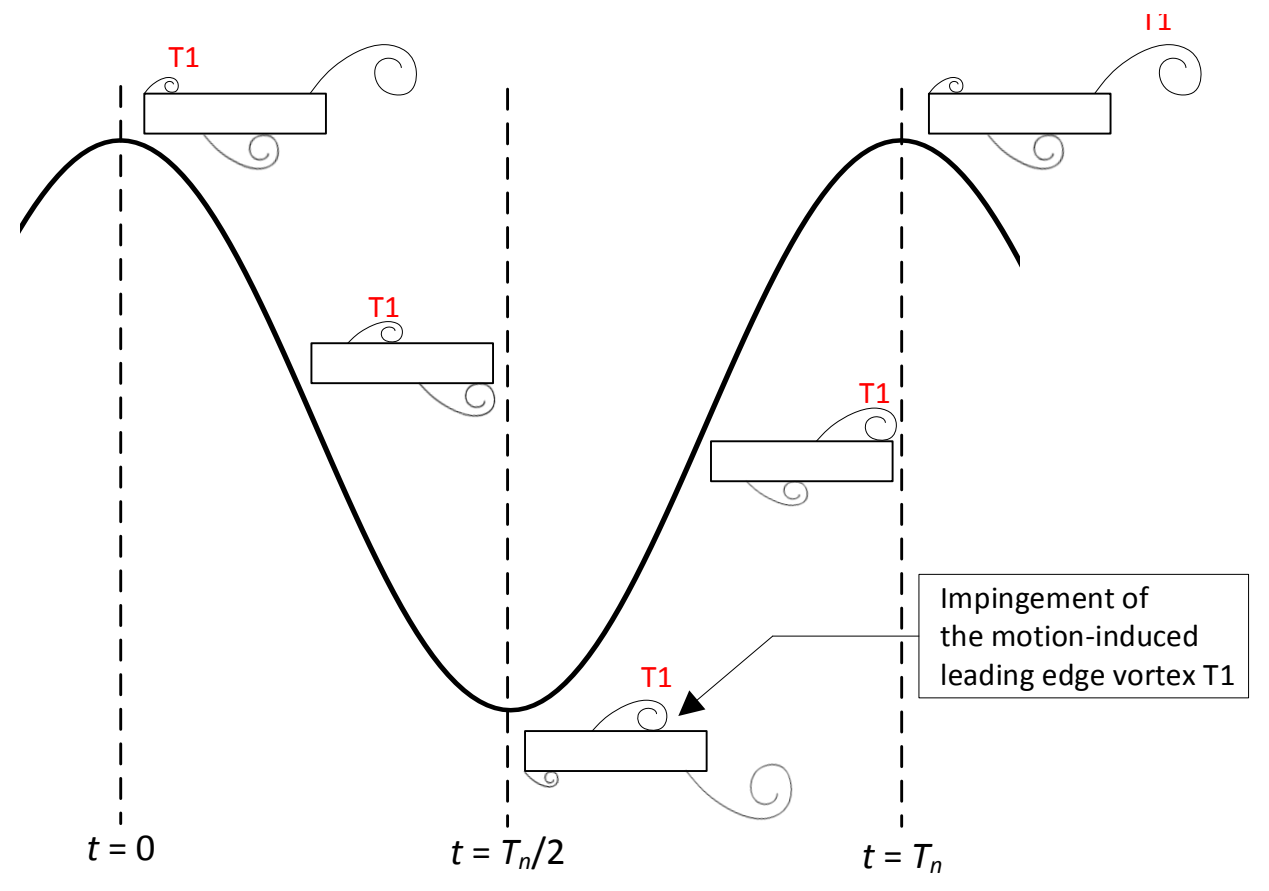

Figure 18: Schematic illustrating the development of the motion-induced leading edge vortex T1 throughout one cycle of the heaving motion during the VIV lock-in.

\subsection{Pitching VIV}

402 When the model was restrained to the pitching mode only, two different behaviours 403 were observed in Figure 19. The torsional flutter occurred at a high wind speed and was 404 characterised by a dramatic increase in the angular displacement. One pitching VIV lock-in was observed at the reduced wind speed $U_{R}=1.03$. The phase analysis of the surface pressure on the top surface revealed there were 1.5 vortices during one cycle of motion (Figure 20) or, in other words, it took 1.5 cycles of motion for one vortex created at the leading edge to reach the trailing edge and then be shed into the wake. Based on Matsumoto et al. (2008), this corresponded to the secondary VIV peak; the primary VIV peak did not appear as was also found by Nakamura and Nakashima (1986). In comparison to the heaving response, as the wind speed increased, the angular response was seen to rise quite suddenly and, beyond the peak, it only gradually decreased. Analysing the phase shift of the moment against the angular displacement revealed a more gradual change in the phase angle during the lock-in. 
(a)

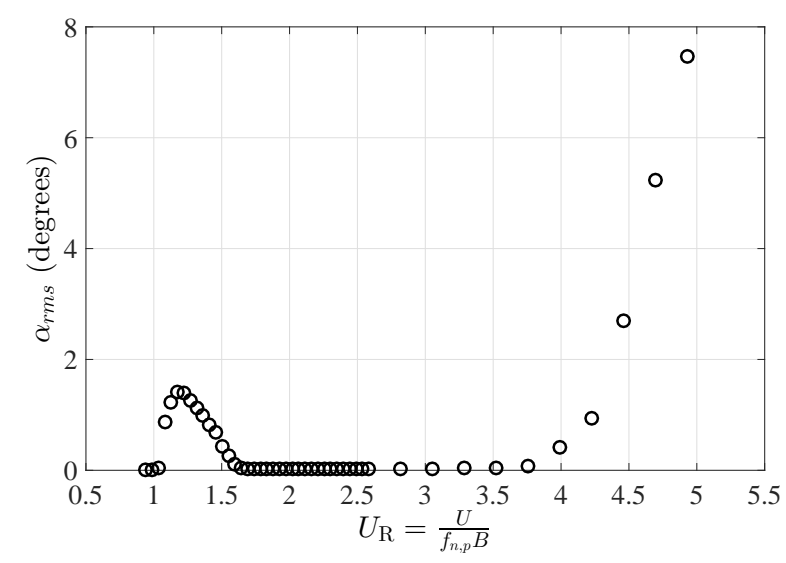

(c)

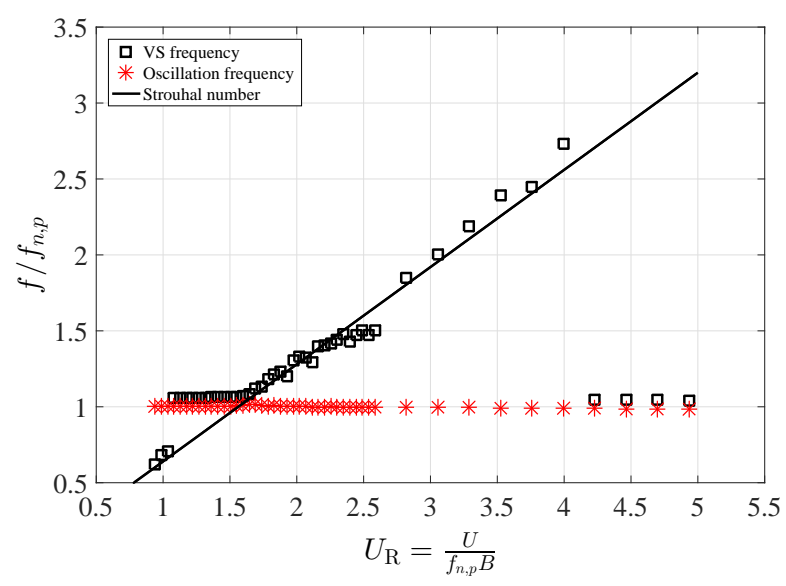

(b)

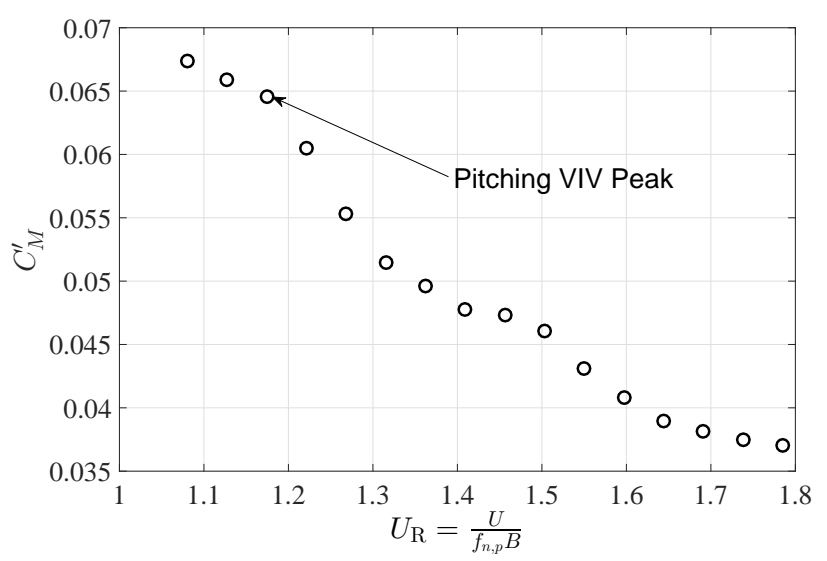

(d)

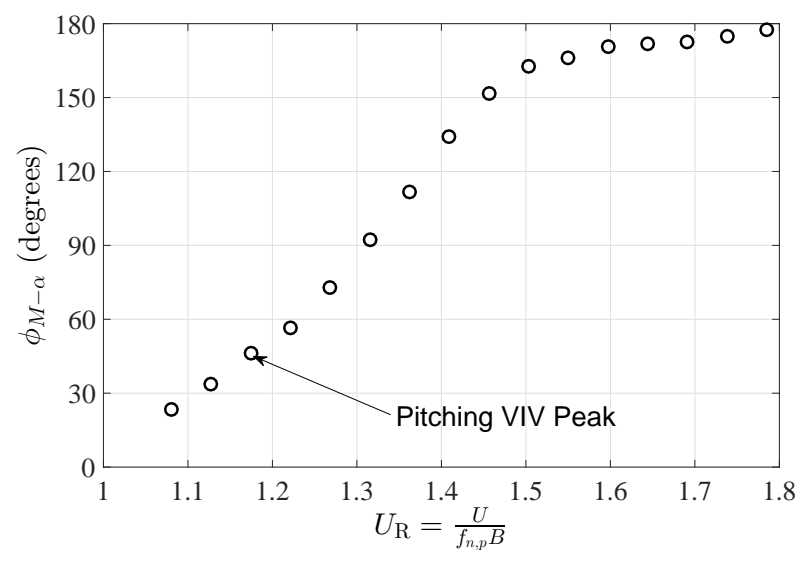

Figure 19: Results of the wind tunnel dynamic test of the section model restrained to the pitching mode only: (a) structural response, (b) moment coefficient response, (c) frequency of response and (d) phase shift of the moment against the structural angular displacement. 


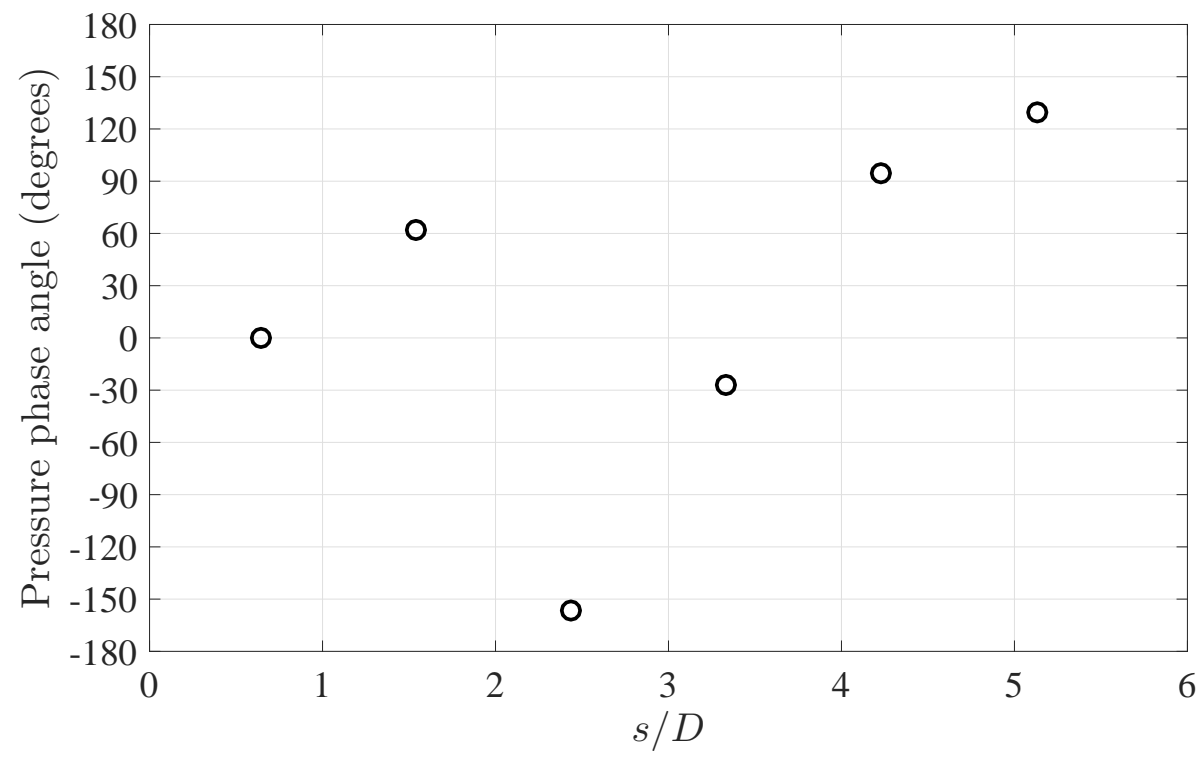

Figure 20: Phase angles of vortices rolling on the surface of the cylinder experiencing the pitching VIV response, measured in the wind tunnel dynamic test at $U_{R}=1.17$, i.e. at the pitching VIV peak.

The variation of the surface pressure correlation measured along the leading edge (Positions $\mathrm{A}$ and $\mathrm{B}$ ) and along the trailing edge (Positions $\mathrm{C}$ and $\mathrm{D}$ ) during the pitching VIV lock-in is summarised in Figure 21. In comparison to what was observed when the cylinder was restrained to the heaving mode only, certain similarities can be drawn. After the maximum structural response was reached, a reduction in the pressure correlation occurred at Position $\mathrm{C}$ and led to a decrease in the amplitude of the structural response. Knowing the phase shift between the surface pressure and the angular displacement, the development of the flow field around the cylinder during two successive cycles of the motion is illustrated in Figure 22. After one cycle of motion, the motion-induced leading-edge vortex travelled downstream a distance up to two-thirds of the width of the cylinder. In the next quarter of the cycle, the upward accelerating motion of the trailing edge caused this vortex to impinge on the surface, leading to a rise in the moment acting on the cylinder. Afterwards, the motion of the cylinder slowed down; the vortex was pushed towards the trailing edge and eventually shed into the wake. This result highlighted the different role of the motion-induced leading-edge vortex and its impingement in the VIV of the 5:1 rectangular cylinder. 
(a) $U_{R}=1.13$

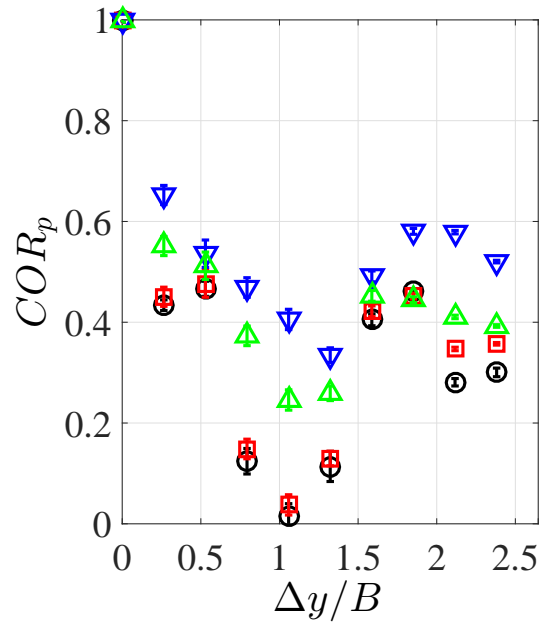

(b) $U_{R}=1.22$

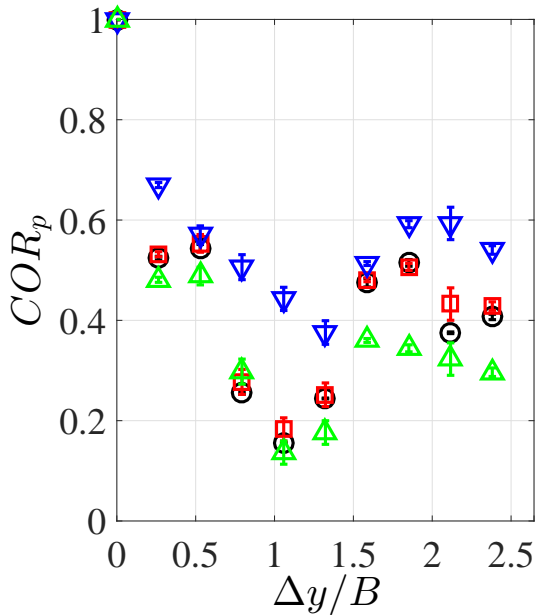

(c) $U_{R}=1.60$

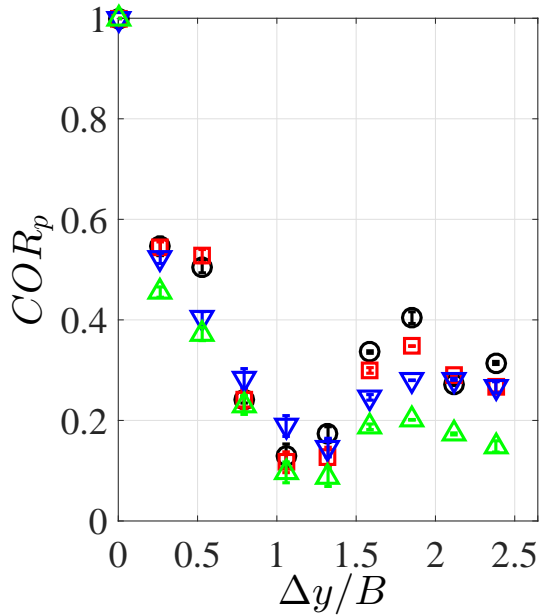

Figure 21: Wind tunnel results of the span-wise pressure correlation measured at 4 stream-wise positions in the smooth flow during the pitching VIV lock-in; black: Position A; red: Position B; blue: Position C; green: Position D.

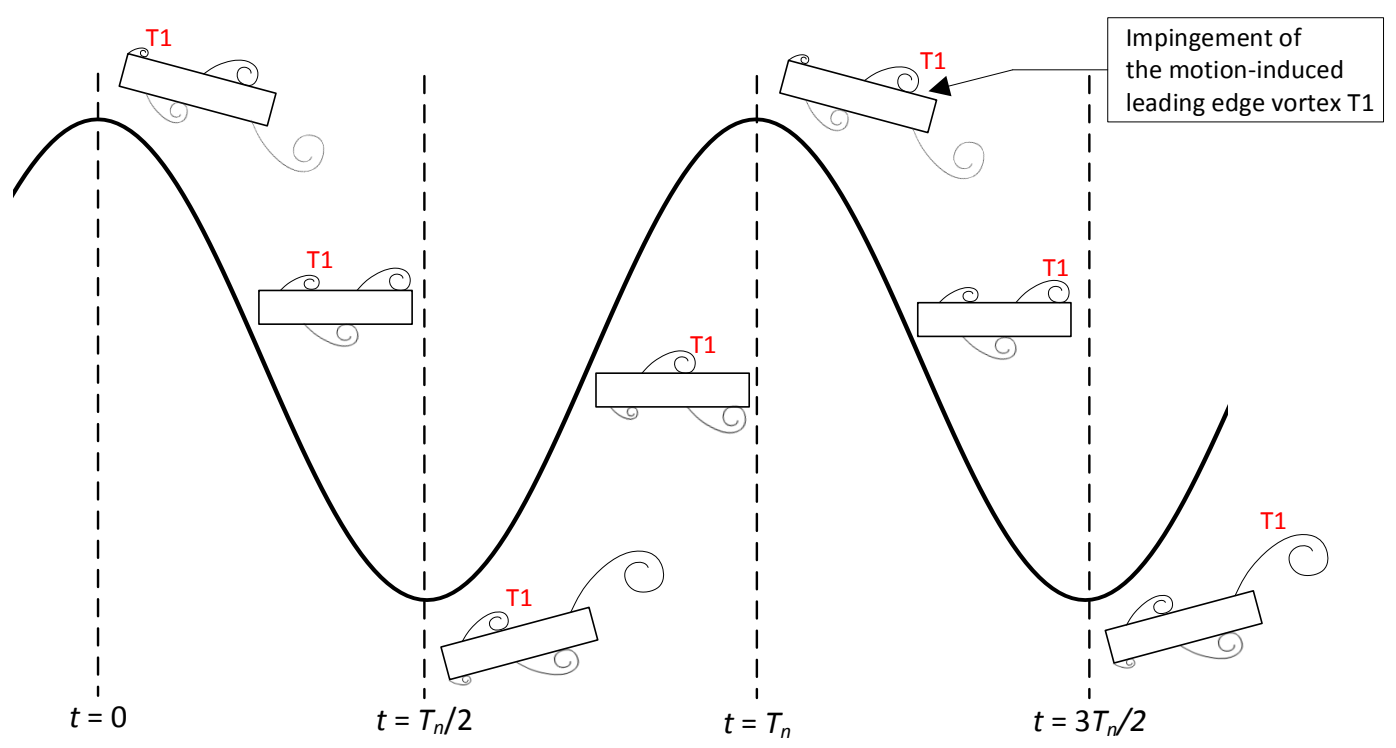

Figure 22: Schematic illustrating the development of the motion-induced leading-edge vortex T1 throughout 1.5 cycles of the pitching motion during the VIV lock-in. 


\section{Conclusion}

430 By analysing the surface pressure correlation along the leading edge and trailing edge 431 and investigating the flow field offered by the computational simulation, this paper presents a 432 comprehensive explanation of the VIV mechanism of the 5:1 rectangular cylinder. Regardless 433 of being restrained to the heaving mode only or the pitching mode only, there were two 434 key flow features which were important for the VIV of this particular geometry. The first 435 one was the leading-edge vortex, which was responsible for triggering the motion, resulting 436 in some initial structural displacement at the start of the lock-in. The second one was 437 the impingement of the motion-induced leading-edge vortex on the surface of the cylinder, 438 occurring close to the trailing edge. This flow feature led to a rise in the suction and in the lift 439 force or moment acting on the cylinder, causing an increase in the structural response during 440 the lock-in. As part of a boarder wind tunnel and computational studies, these outcomes 441 will be analysed to provide more insight into the turbulence-induced effect of the VIV of a 442 5:1 rectangular cylinder; further results and discussion will be presented in a separate paper.

443 There were a number of limitations to the work presented in this paper. As for CFD 444 simulations, the use of finer computational domains particularly in the span-wise direction is 445 of importance to minimise issues related to the mesh sensitivity. In addition, experimental 446 errors were observed in WT dynamic tests; the combination of the end plates, the finite span447 wise length of the model and its rolling oscillation caused some resonance effect limiting the 448 usability of the pressure data to investigate the span-wise correlation. This issue should be 449 studied and a standard guideline to perform dynamic wind tunnel tests should be produced

\section{Acknowledgements}

The work presented here was supported by the University of Nottingham through the Dean of Engineering Research Scholarship for International Excellence, the access to the Atmospheric Boundary Layer wind tunnel and the High Performance Computer. The authors wish to express their sincere thanks to technicians in the Department of Civil Engineering during wind tunnel tests.

\section{References}

Bai, Y., Yang, K., Sun, D., Zhang, Y., Kennedy, D., Williams, F., Gao, X., 2013. Numerical aerodynamic analysis of bluff bodies at a high Reynolds number with three-dimensional CFD modelling. Science China: Physics, Mechanics and Astronomy 56, 277 - 289.

Batina, J., 1990. Unsteady Euler airfoil solutions using unstructured dynamic meshes. AIAA Journal 28 (8), $1381-1388$.

Bruno, L., Coste, N., Fransos, D., 2011. Simulated flow around a rectangular 5:1 cylinder: Spanwise discretisation effects and emerging flow features. Journal of Wind Engineering and Industrial Aerodynamics 104 - 106 (0), 203 - 215. 
465

466

467

468

469

470

471 472

473

474

475

476 477

478

480

481 482 483

484 485

486 487 488 489

490 491 492

493 494 495

496 497 498

499 500 501

Bruno, L., Fransos, D., Coste, N., Bosco, A., 2010. 3D flow around a rectangular cylinder: A computational study. Journal of Wind Engineering and Industrial Aerodynamics 98, 263 $-276$.

Bruno, L., Salvetti, M., Ricciardelli, F., 2014. Benchmark on the Aerodynamics of a Rectangular 5:1 Cylinder: An overview after the first four years of activity. Journal of Wind Engineering and Industrial Aerodynamics 126 (0), 87 - 106.

Cao, S., 2015. Towards better understanding of bridge aerodynamics - turbulence effects. In: The $14^{\text {th }}$ International Conference on Wind Engineering. Porto Alegre, Brazil.

Celik, I., Ghia, U., Roaches, P., Freitas, C., Coleman, H., Raad, P., 2008. Procedure for estimation and reporting of uncertainty due to discretisation in CFD applications. Journal of Fluids Engineering 130 (7).

Celik, I. B., Cehreli, Z. N., Yavuz, I., 2005. Index of resolution quality for Large Eddy Simulation. Journal of Fluids Engineering 127 (5), 949 - 958.

Daniels, S., Castro, I., Xie, Z., 2014. Free-stream turbulence effects on bridge decks undergoing vortex-induced vibrations using Large-Eddy Simulation. In: The $11^{\text {th }}$ UK Conference on Wind Engineering. Birmingham, UK.

Furby, C., Tabor, G., Weller, H., Gosman, A., 1997. A comparative study of subgrid scale models in homogeneous isotropic trubulence. Physics of Fluids (1994-present) 9 (5), 1416 -1429 .

Goswami, I., Scanlan, R., Jones, N., 1993. Vortex-induced vibration of circular cylinder. Journal of Engineering Mechanics 11, 2270 - 2287.

Kareem, A., Wu, T., 2013. Wind-induced effects on bluff bodies in turbulent flows: Nonstationary, non-Gaussian and nonlinear features . Journal of Wind Engineering and Industrial Aerodynamics $122(0), 21$ - 37, the Seventh International Colloquium on Bluff Body Aerodynamics and Applications (BBAA7).

Kawatani, M., Kim, H., Uejima, H., Kobayashi, H., 1993. Effects of turbulence flows on vortex-induced oscillation of bridge girders with basic sections. Journal of Wind Engineering and Industrial Applications 49 (1 - 3), 477 - 486.

Kawatani, M., Toda, N., Sato, M., Kobayashi, H., 1999. Vortex-induced torsional oscillations of bridge girders with basic sections in turbulent flows. Journal of Wind Engineering and Industrial Applications 83 (1 - 3), 327 - 336.

Kobayashi, H., Kawatani, M., Kim, H., 1992. Effects of turbulence characteristics on vortexinduced oscillation of rectangular cylinders. Journal of Wind Engineering and Industrial Applications 41, $775-784$.

Kobayashi, H., Kawatani, M., Nakade, O., 1990. Vortex-induced oscillations of two dimensional rectangular cylinders in large scale turbulence. Journal of Wind Engineering and Industrial Applications 33 (1 - 2), 101 - 106. 
502 Larsen, A., Walther, J., 1998. Discrete vortex simulation of flow around five generic bridge deck sections. Journal of Wind Engineering and Industrial Aerodynamics $7 \mathbf{7}$ - 78, 591 602 .

Lee, B., 1975. The effects of turbulence on the surface pressure field of a square prism. Journal of Fluid Mechanics 69 (2), $263-282$.

Mannini, C., Soda, A., Schewe, G., 2011. Numerical investigation on the three-dimensional unsteady flow past a 5:1 rectangular cylinder. Journal of Wind Engineering and Aerodynamics 99 (4), $469-482$.

Matsumoto, M., Shiraishi, N., Shirato, H., Stoyanoff, S., Yagi, T., 1993. Mechanism of, and turbulence effect on vortex-induced oscillations for bridge box girders. Journal of Wind Engineering and Industrial Aerodynamics 49 (1 - 3), 467 - 478.

Matsumoto, M., Shirato, H., Araki, K., Haramura, T., Hashimoto, T., 2003. Spanwise coherence characteristics of surface pressure field on 2D bluff bodies. Journal of Wind Engineering and Industrial Aerodynamics 91 (1 - 2), 155 - 163.

Matsumoto, M., Yagi, T., Tamaki, H., Tsubota, T., 2008. Vortex-induced vibration and its effect on torsional flutter instability in the case of $B / D=4$ rectangular cylinder. Journal of Wind Engineering and Industrial Aerodynamics 96 (6-7), $971-983$.

Mills, R., Sheridan, J., Hourigan, K., 2003. Partical Image Velocimetry and visualisation of natural and forced flow around rectangular. Journal of Wind and Mechanics 478, 299 323.

Nakamura, Y., Nakashima, M., 1986. Vortex excitation of prisms with elongated rectangular, $\mathrm{H}$ and T-cross-sections. Journal of Fluids and Structures 163, 149 - 169.

Nakamura, Y., Ohya, Y., Tsuruta, H., 1991. Experiments on vortex shedding from flat plates with square leading and trailing edges. Journal of Fluid Engineering 222, 473 - 447.

Ohya, Y., Nakamura, Y., Ozono, S., Tsuruta, H., 1992. A numerical study of vortex shedding from flat plates with square leading and trailing edges. Journal of Fluid Mechanics 236, $445-460$.

Owen, J., Hargreaves, D., Gu, X., 2006. Modelling the Mechanism of Vortex Induced Response of Bridge Deck. In: The $7^{\text {th }}$ UK Conference on Wind Engineering (WES 06). Glasgow.

Ozono, S., Ohya, Y., Nakamura, Y., Nakayama, R., 1992. Stepwise increase in the Strouhal number for flows around flat plates. International Journal for Numerical Methods in Fluid 15 (9), $1025-1036$.

Ricciardelli, F., Marra, A., 2008. Sectional aerodynmic forces and their longitudinal correlation on a vibrating 5:1 rectangular cylinder. In: BBAA VI International Colloquium on: Bluff Bodies Aerodynamics and Applications. Milano, Italy.

Roache, P., 1997. Quantification of uncertainty in computational fluid dynamics. Annual Reviews of Fluid Mechanics 29, 123 - 160. 
539 Schewe, G., 2013. Reynolds-number-effect in flow around a rectangular cylinder with aspect $540 \quad$ ratio 1:5. Journal of Fluids and Structures 36 (0), $16-25$.

541 Sun, D., Owen, J., Wright, N., 2009. Application of the k- $\omega$ turbulence model for a wind542 induced vibration study of 2D bluff bodies. Journal of Wind Engineering and Industrial 543 Aerodynamics 97 (2).

544 Sun, D., Owen, J., Wright, N., Liaw, K., 2008. Fluid-structure interaction of prismatic line545 like structure, using LES and block-iterative coupling. Journal of Wind Engineering and $546 \quad$ Industrial Aerodynamics $96(6-7)$.

547 Tan, B., Thompson, M., Hourigan, K., 1998. Simulated flow around long plates under cross 548 flow pertubations. International Journal of Fluid Dynamics 2 (1).

549 Waterson, N., Baker, N., 2010. Numerical Prediction of Flutter Behaviour for Long-span 550 Bridge Decks. In: The $5^{\text {th }}$ International Symposium on Computational Wind Engineering 551 (CWE2010). North Carolina, USA.

552 Wu, T., Kareem, A., 2012. An overview of vortex-induced vibration (VIV) of bridge decks. 553 Journal of Frontiers of Structural and Civil Engineering 6 (64), 335 - 347.

554 Xiang, H., Ge, Y., 2002. Refinements on aerodynamic stability analysis of super long-span 555 bridge. Journal of Wind Engineering and Industrial Aerodynamics 90 (12 - 15), 1493 556 1515, fifth Asia-Pacific Conference on Wind Engineering.

557 Zhu, Z., Chen, Z., 2013. Large eddy simulation of aerodynamics of a flat box girder on 558 long-span bridges. Procedia Engineering 61, 212 - 219. 